\title{
Representation of Banach Ideal Spaces and Factorization of Operators
}

\author{
Evgenii I. Berezhnoĭ and Lech Maligranda
}

Abstract. Representation theorems are proved for Banach ideal spaces with the Fatou property which are built by the Calderón-Lozanovskiu construction. Factorization theorems for operators in spaces more general than the Lebesgue $L^{p}$ spaces are investigated. It is natural to extend the Gagliardo theorem on the Schur test and the Rubio de Francia theorem on factorization of the Muckenhoupt $A_{p}$ weights to reflexive Orlicz spaces. However, it turns out that for the scales far from $L^{p}$-spaces this is impossible. For the concrete integral operators it is shown that factorization theorems and the Schur test in some reflexive Orlicz spaces are not valid. Representation theorems for the Calderón-Lozanovskiı construction are involved in the proofs.

\section{Introduction}

Let $\left(X_{0}, X_{1}\right)$ be a compatible couple of Banach spaces and let $F\left(X_{0}, X_{1}\right)$ be a Banach space intermediate between $X_{0}$ and $X_{1}$ generated by an construction $F$ (maybe interpolation one). The so-called inverse interpolation problem is the problem whether the space $F\left(X_{0}, X_{1}\right)$ may be obtained by the same functor $F$ from other compatible Banach couples having some additional properties.

Consider also a uniqueness problem for the fixed construction or interpolation method $F$, that is, the following problem: Does the equality $F\left(X_{0}, X_{1}\right)=F\left(Y_{0}, Y_{1}\right)$ with equivalent norms for arbitrary Banach spaces $X_{0}, X_{1}, Y_{0}, Y_{1}$, or for the spaces from a given class, imply that $X_{0}=Y_{0}$ and $X_{1}=Y_{1}$ with equivalence of their norms?

The above two problems are difficult to solve, therefore it makes sense to consider their simpler versions. In this paper we consider a uniqueness problem for the fixed construction or interpolation method $F$, sometimes also called the representation of the space $F(\cdot)$, by asking the following question:

Does the equality $F\left(X_{0}, X_{1}\right)=F\left(X_{0}, X_{2}\right)$ hold with equivalent norms for arbitrary Banach spaces $X_{0}, X_{1}, X_{2}$, or for the spaces from a given class, imply that $X_{1}=X_{2}$ with equivalent norms?

The non-uniqueness of the real and the complex method are well known. Already, Grisvard, Seeley and others (see [53], pages 320-321 for references) have considered

Received by the editors September 2, 2003; revised April 23, 2004.

Research supported by a grant from the Royal Swedish Academy of Sciences for cooperation between Sweden and the former Soviet Union (projects 35146 and 35155). The first author was also supported by the Russian Fond of Fundamental Investigations-grant 02-01-00428. The second author was also supported by the Swedish Natural Science Research Council (NFR)-grant M5105-20005228/2000.

AMS subject classification: 46E30, 46B42, 46B70.

Keywords: Banach ideal spaces, weighted spaces, weight functions, Calderón-Lozanovskiĭ spaces, Orlicz spaces, representation of spaces, uniqueness problem, positive linear operators, positive sublinear operators, Schur test, factorization of operators, factorization of weights, complex interpolation method, real interpolation method.

(C) Canadian Mathematical Society 2005. 
real and complex interpolation with boundary conditions and proved that for $0<$ $\theta<1 / 2$,

$$
\left[L^{2}(\Omega), W^{1,2}(\Omega)\right]_{\theta}=\left[L^{2}(\Omega), W_{0}^{1,2}(\Omega)\right]_{\theta}=W^{\theta, 2}(\Omega),
$$

where $\Omega \subset R^{n}$ is a bounded $C^{\infty}$-domain and $W_{0}^{\theta, 2}(\Omega)=\left\{x \in W^{\theta, 2}(\Omega): u_{\mid \partial \Omega}=0\right\}$, with $W^{\theta, 2}$ being the usual Sobolev space.

For the real interpolation construction, Lions-Magenes (see [28, Theorem 11.1, p. 55]) proved that for $0<\theta<1 / 2$,

$$
\left(L^{2}(\Omega), W^{1,2}(\Omega)\right)_{\theta, 2}=\left(L^{2}(\Omega), W_{0}^{1,2}(\Omega)\right)_{\theta, 2}=W^{\theta, 2}(\Omega),
$$

where $\Omega=\left\{(x, y): x^{2}+y^{2}<1\right\}$. For the real interpolation method we can also take Triebel's example connected with the negative answer for the problem of interpolation of intersections. For the weight function $w(t)=\min (t, 1-t)^{-1 / 2}, t \in(0,1)$ and the spaces on $(0,1)$ we have for $1 / 2<\theta<1$,

$$
\left(L^{2}, W^{1,2} \cap L_{w}^{2}\right)_{\theta, 2}=\left(L^{2}, W_{0}^{1,2}\right)_{\theta, 2}=W_{0}^{\theta, 2},
$$

where $W_{0}^{\theta, 2}$ is the closure of $C_{0}^{\infty}(0,1)$ in $W^{\theta, 2}$. Moreover, Wallsten has given an example of a space $M\left(c f\right.$. [54]) for which $\left(M, L^{\infty}\right)_{\theta, p}=\left(L^{1}, L^{\infty}\right)_{\theta, p}$ for $1 / 2<\theta<1$.

In the seventies, Fefferman-Stein, Rivière-Sagher, Hanks, Bennett-Sharpley and others (see $[2, \S 5.6-5.7]$ for results and references) proved equalities for the complex interpolation method:

$$
\left[H^{1}, L^{p}\right]_{\theta}=L^{q}=\left[L^{1}, L^{p}\right]_{\theta} \text { and }\left[L^{p}, \mathrm{BMO}\right]_{\theta}=L^{\frac{p}{1-\theta}}=\left[L^{p}, L^{\infty}\right]_{\theta}
$$

for all $0<\theta<1,1<p<\infty$ and $1 / q=1-\theta+\theta / p$, and for the real interpolation method:

$$
\begin{aligned}
\left(L^{1}, L^{\infty}\right)_{\theta, p} & =\left(\operatorname{Re} H^{1}, L^{\infty}\right)_{\theta, p}=\left(L^{1}, \mathrm{BMO}\right)_{\theta, p} \\
& =\left(\operatorname{Re} H^{1}, L^{\infty}\right)_{\theta, p}=\left(\operatorname{Re} H^{1}, \mathrm{BMO}\right)_{\theta, p}=L^{\frac{1}{1-\theta}, p}
\end{aligned}
$$

for all $0<\theta<1,1 \leq p \leq \infty$, where $L^{\frac{1}{1-\theta}, p}$ are classical Lorentz spaces. We also mention that for an arbitrary couple of Banach spaces $X_{0}$ and $X_{1}$, the equalities

$$
\left[\tilde{X}_{0}, \tilde{X}_{1}\right]_{\theta}=\left[X_{0}, X_{1}\right]_{\theta}=\left[X_{0}^{0}, X_{1}^{0}\right]_{\theta} \text { and }\left(\tilde{X}_{0}, \tilde{X}_{1}\right)_{\theta, p}=\left(X_{0}, X_{1}\right)_{\theta, p}
$$

hold isometrically for all $0<\theta<1,1 \leq p \leq \infty$, where $\tilde{X}_{i}$ is the Gagliardo completion of $X_{i}$ and $X_{i}^{0}$ is the closure of $X_{0} \cap X_{1}$ in $X_{i}$ for $i=0,1$.

Let us point out that the situation may be quite different if we assume from the beginning that all the spaces in the problem have some common structure, for example, they are Banach lattices on a given measure space (Banach ideal spaces). This phenomenon, that some problem has negative solution for general Banach spaces but positive answer in the class of ideal Banach spaces with the Fatou property, was first observed in $[33,34]$ in connection to Peetre's problem on interpolation of intersections. 
Cwikel and Nilsson [14] showed the uniqueness theorem for the Calderón construction $F\left(X_{0}, X_{1}\right)=X_{0}^{1-\theta} X_{1}^{\theta}$ when $X_{0}, X_{1}, X_{2}$ are Banach ideal spaces and all have the Fatou property. Their arguments used in the proof are related to ideas in a theorem of Pisier [45]. Some related results for finite dimensional Banach spaces were considered by Rochberg [47].

The inverse interpolation problem for the real method of interpolation $F=(\cdot)_{\Phi}$ on some class of Banach ideal spaces was investigated in [5]. One of the results shows that if $X$ is a symmetric space on $(0, \infty)$ and $\left(X, L^{\infty}\right)_{\theta, p}=\left(L^{p}, L^{\infty}\right)_{\theta, p}$, then the fundamental function of $X$ is equivalent to $t^{1 / p}$.

Cwikel-Nilsson [14, p. 45] and Asekritova-Krugljak [1, p. 114] proved the following uniqueness theorem for the real interpolation method:

Let $X_{0}, X_{1}, Y_{0}, Y_{1}$ be Banach ideal spaces. If $\left(X_{0}, X_{1}\right)_{\theta_{i}, p_{i}}=\left(Y_{0}, Y_{1}\right)_{\theta_{i}, p_{i}}, i=0,1$, for some $\theta_{0}, \theta_{1} \in(0,1), \theta_{0} \neq \theta_{1}$ and $p_{0}, p_{1} \in[1, \infty]$, then $\left(X_{0}, X_{1}\right)_{\theta, p}=\left(Y_{0}, Y_{1}\right)_{\theta, p}$ for all $\theta \in(0,1)$ and $p \in[1, \infty]$.

In this paper we consider uniqueness results (representation theorems) for the Calderón-Lozanovskiĭ construction $F\left(X_{0}, X_{1}\right)=\varphi\left(X_{0}, X_{1}\right)$ with a general function $\varphi \in \mathcal{U}$.

These results have applications in the factorization of operators between Banach ideal spaces. In the theory of integral operators with positive kernels a special role is played by the so-called Schur lemma or Schur test (see [23, p. 37] or [52, p. 42]), which says that an integral operator $K x(t)=\int k(t, s) x(s) d s$ with a positive kernel $k(t, s) \geq 0$ is bounded in $L^{p}$ for $1<p<\infty$ if and only if there exists a positive function $u$ such that

$$
K u^{p^{\prime}}(t) \leq C u^{p^{\prime}}(t) \text { and } K^{\prime} u^{p}(t) \leq C u^{p}(t),
$$

where $K^{\prime}$ is a formally associate operator and $1 / p^{\prime}+1 / p=1$. We can rewrite this in the factorization way: there exists a positive function $u$ (weight function $u$ ) such that

$$
K: L_{u^{p}}^{1} \rightarrow L_{u^{p}}^{1} \text { and } K: L_{u^{-p^{\prime}}}^{\infty} \rightarrow L_{u^{-p^{\prime}}}^{\infty} \text { is bounded. }
$$

In the eighties, interest in statements like the Schur lemma increased after the solution of the factorization problem of Muckenhoupt's $A_{p}$-condition on weight by Jones [21], and even stronger after the Rubio de Francia elementary proof of Jones' theorem $[13,48]$. These studies were later developed in $[9,12,16-18,20,49]$. All these papers contain the factorization problem of various classical operators in weighted $L^{p}$ spaces.

We will extend factorization theorems from weighted $L^{p}$ spaces to weighted Banach ideal $X^{(p)}$ spaces, and the factorization will be proved first through the weighted $L^{\infty}$ spaces. The main factorization problem is to have factorization through the weighted $L^{1}$ and weighted $L^{\infty}$ spaces and this question will be also discussed here.

We prove the factorization result for a sufficiently large class of positive sublinear bounded operators $T$ between $L^{p}$ spaces through the Lorentz and Marcinkiewicz spaces generated by a certain weight function. Then we show that factorization of the symmetric space $X^{(p)}$ through weighted $X$ and $L^{\infty}$ is not true for the positive sublinear Hardy operator. 
The failure of the main factorization theorem in Calderón-Lozanovskii spaces generated by a non-power function is proved for the Volterra operator and the averaging operator. This shows that we cannot go far from the scale of $L^{p}$ spaces with the factorization theorems.

Finally, we show that the Schur lemma is not true in some reflexive Orlicz spaces for the classical Hardy operator; that is, we can construct reflexive Orlicz spaces in which the classical Hardy operator is bounded (it is bounded even in any reflexive Orlicz space) but the factorization through weighted $L^{1}$ and weighted $L^{\infty}$ spaces is not possible.

Let us mention that a quite different question, called also the Lions problem, about the effective dependence of a given family of spaces on its function parameter $\varphi$, was considered for the complex method of interpolation by Stafney [51], for the real method of interpolation in $[1,8,19]$, for the Calderón-Lozanovskii construction $\varphi(\cdot)$ in [6], and for the Gustavsson-Peetre construction $G_{\varphi}(\cdot)$ in [7]. The question for the Calderón-Lozanovskiu construction is: when are the spaces $\varphi_{0}\left(X_{0}, X_{1}\right)$ and $\varphi_{1}\left(X_{0}, X_{1}\right)$ different for $\varphi_{0} \neq \varphi_{1}$ ?

The content of the paper is as follows: In Section 1 we define the Banach ideal spaces and the Calderón-Lozanovskii construction and collect their properties including the Lozanovskii factorization theorem.

In Section 2 we prove representation theorems, called also uniqueness theorems, for the Calderón-Lozanovskii construction generated by different Banach ideal spaces or the weighted $L^{p}$ spaces. The main representation theorems (Theorems 1-4) show that under some little assumption on $\varphi$ the equality $\varphi\left(X_{0}, X_{1}\right)=\varphi\left(X_{0}, X_{2}\right)$ with equivalent norms implies that $X_{1}=X_{2}$ with equivalent norms, and the equality $\varphi\left(L_{u}^{1}, L_{v}^{\infty}\right)=\varphi\left(L_{w}^{1}, L^{\infty}\right)$ with equivalent norms implies the equivalence of weights $w^{\theta} \approx u^{\theta} v^{1-\theta}$ for some $0 \leq \theta \leq 1$.

Section 3 contains pointwise estimates for positive sublinear operators. Factorization results in weighted $X^{(p)}$ spaces are presented there. They are extensions of the corresponding results of Hernández $[17,18]$ for weighted $L^{p}$ spaces. The main tool in the proofs is Lemma 6 of the Gagliardo and Rubio de Francia type.

For a large class of positive sublinear operators $T$ which are bounded between $L^{p}$ spaces we show a factorization of $T$ through the Lorentz and Marcinkiewicz spaces generated by a certain weight function.

We also prove that the positive sublinear Hardy operator bounded between symmetric spaces $X^{(p)}$ cannot be factorized by a weighted space $X$ and weighted $L^{\infty}$ when the upper Boyd index of the space $X$ is 1 . This example of the Hardy positive sublinear operator shows that without any additional assumptions on an operator the factorization theorem through weighted $L^{1}$ and weighted $L^{\infty}$ spaces cannot be true.

In Section 4, representation theorems are used to show that the factorization problem in Calderón-Lozanovskii spaces generated by a non-power function is not true, in general, for the Volterra operator and in Section 5 the same is done for the averaging operator.

Section 6 contains a counter-example showing that the classical Hardy operator between some reflexive Orlicz spaces cannot be factorized through weighted $L^{1}$ and weighted $L^{\infty}$ spaces. This also shows that the Schur lemma for positive integral operators between some reflexive Orlicz spaces is false. Detailed proofs of the construc- 
tions of the functions in the counter-example are collected in an appendix.

Preliminary versions of Theorems 4, 8 and 9 were announced without proofs in [4].

\section{Banach Ideal Spaces and the Calderón-Lozanovskii Construction}

Let $(\Omega, \mu)$ be a complete $\sigma$-finite measure space and let $L^{0}(\mu)$ or $L^{0}(\Omega)$ denote, as usual, the space of all equivalence classes of measurable functions on $\Omega$ with the topology of convergence in measure on $\mu$-finite sets. The order $|x| \leq|y|$ means that $|x(t)| \leq|y(t)|$ for $\mu$-almost all $t \in \Omega$.

A Banach subspace $X=\left(X,\|\cdot\|_{X}\right)$ of $L^{0}(\mu)$ such that there exists $u \in X$ with $u>0 \mu$-a.e. on $\Omega$ and $\|x\|_{X} \leq\|y\|_{X}$ whenever $|x| \leq|y|$ is called a Banach ideal space on $\Omega$ or on $(\Omega, \mu)$.

If $X$ is a Banach ideal space on $\Omega$ and $w \in L^{0}(\mu)$ is a weight function on $\Omega$, that is, $w>0$ a.e. on $\Omega$, we define the weighted space $X_{w}$ by $\|x\|_{X_{w}}:=\|x w\|_{X}$.

The associated space $X^{\prime}$ to $X$ is the space of all $x \in L^{0}(\mu)$ such that

$$
\int_{\Omega}|x(t) y(t)| d \mu<\infty
$$

for every $y \in X$ endowed with the norm

$$
\|x\|_{X^{\prime}}=\sup \left\{\int_{\Omega}|x(t) y(t)| d t:\|y\|_{X} \leq 1\right\} .
$$

$X^{\prime}$ is a Banach ideal space.

A Banach ideal space $X$ with a norm $\|\cdot\|_{X}$ has the Fatou property if for any increasing positive sequence $\left(x_{n}\right)$ in $X$ with $\sup _{n}\left\|x_{n}\right\|_{X}<\infty$ we have that $\sup _{n} x_{n} \in X$ and $\left\|\sup _{n} x_{n}\right\|_{X}=\sup _{n}\left\|x_{n}\right\|_{X}$.

For every Banach ideal space $X$ we have the embedding $X \subset X^{\prime \prime}$ with $\|x\|_{X^{\prime \prime}} \leq$ $\|x\|_{X}$ for any $x \in X$. Moreover, $X=X^{\prime \prime}$ with equality of the norms if and only if $X$ has the Fatou property $(c f .[25,27])$.

Let $\bar{X}=\left(X_{0}, X_{1}\right)$ be a couple of Banach ideal spaces on $\Omega$ and let $\mathcal{U}$ denote the set of all non-negative, concave and positively homogeneous continuous functions $\varphi:[0, \infty) \times[0, \infty) \rightarrow[0, \infty)$ such that $\varphi(0,0)=0$. Then the Calderón-Lozanovskiu construction or the Calderón-Lozanovskiu spaces $\varphi(\bar{X})=\varphi\left(X_{0}, X_{1}\right)$ consists of all $x \in$ $L^{0}(\mu)$ such that $|x| \leq \lambda \varphi\left(\left|x_{0}\right|,\left|x_{1}\right|\right)$ for some $x_{i} \in X_{i}$ with $\left\|x_{i}\right\|_{X_{i}} \leq 1, i=0,1$. The spaces $\varphi(\bar{X})$ are Banach ideal spaces on $\Omega$ equipped with the norm

$$
\|x\|_{\varphi}=\inf \left\{\lambda>0 ;|x| \leq \lambda \varphi\left(\left|x_{0}\right|,\left|x_{1}\right|\right),\left\|x_{0}\right\|_{X_{0}} \leq 1,\left\|x_{1}\right\|_{X_{1}} \leq 1\right\}
$$

(see [30]). In the case of power functions $\varphi_{\theta}(s, t)=s^{1-\theta} t^{\theta}$ with $0 \leq \theta \leq 1, \varphi_{\theta}(\bar{X})$ are the well known Calderón spaces $X_{0}^{1-\theta} X_{1}^{\theta}$ (see [11]). The particular case $X^{\theta}\left(L^{\infty}\right)^{1-\theta}=$ $X^{(p)}$ for $\theta=1 / p, 1 \leq p<\infty$, is known as the $p$-convexification of $X$ (see $\left.[27,38]\right)$.

The properties of $\varphi(\bar{X})$ were studied by Lozanovskil in $[30,31]$ (see also [35]), where among other facts is proved the duality result

$$
\varphi\left(X_{0}, X_{1}\right)^{\prime}=\hat{\varphi}\left(X_{0}^{\prime}, X_{1}^{\prime}\right)
$$


with equivalent norms. Here, for $\varphi \in \mathcal{U}$, the conjugate function $\hat{\varphi}$ is defined by

$$
\hat{\varphi}(s, t):=\inf \left\{\frac{\alpha s+\beta t}{\varphi(\alpha, \beta)}: \alpha, \beta>0\right\}, \quad s, t \geq 0 .
$$

We have $\hat{\varphi} \in \mathcal{U}$ and $\hat{\hat{\varphi}}=\varphi$ (see [31,32] and [35, Lemma 15.8]). Note that

$$
\frac{1}{\varphi\left(\frac{1}{s}, \frac{1}{t}\right)}=\inf _{\alpha, \beta>0} \frac{\max (\alpha s, \beta t)}{\varphi(\alpha, \beta)} \leq \hat{\varphi}(s, t) \leq \frac{2}{\varphi\left(\frac{1}{s}, \frac{1}{t}\right)} .
$$

Lozanovskiu also showed that $\left(X_{0}^{1-\theta} X_{1}^{\theta}\right)^{\prime}=\left(X_{0}^{\prime}\right)^{1-\theta}\left(X_{1}^{\prime}\right)^{\theta}$ with equality of the norms ([29], Theorem 2). Using this equality for $\theta=1 / 2$ it was shown in [29] that $X^{1 / 2}\left(X^{\prime}\right)^{1 / 2}=L^{2}$ isometrically. From this result follows the Lozanovskii factorization theorem, proved in [29, Theorem 6] (see also [35, p. 185] and [46]):

Theorem $A$ Let $X$ be a Banach ideal space. Then for every $0 \leq z \in L^{1}$ and $\varepsilon>0$ we can find $0 \leq x \in X$ and $0 \leq y \in X^{\prime}$ such that $z=x y$ and

$$
\|x\|_{X}\|y\|_{X^{\prime}} \leq(1+\varepsilon)\|z\|_{1} .
$$

If $X$ has the Fatou property, we may take $\varepsilon=0$ in the above inequality.

Calderón-Lozanovskiŭ spaces are closely related to Orlicz spaces. Let $M:[0, \infty) \rightarrow$ $[0, \infty]$ be a nondecreasing, convex and left-continuous function, not identical 0 or $\infty$ on $(0, \infty)$, with $M(0)=0$. Let $\varphi \in \mathcal{U}$ be defined by $\varphi(s, t)=t M^{-1}(s / t)$ if $t>0$ and 0 if $t=0$, where $M^{-1}$ is the right continuous inverse of $M$. Then for any Banach ideal space $X$ on $\Omega$, the Calderón-Lozanovskiu space $\varphi\left(X, L^{\infty}\right)$ is the Banach ideal space

$$
X^{M}=\left\{x \in L^{0}(\mu) ; M(|x| / \lambda) \in X \text { for some } \lambda>0\right\}
$$

equipped with the norm

$$
\|x\|_{X^{M}}=\inf \left\{\lambda>0 ;\|M(|x| / \lambda)\|_{X} \leq 1\right\}
$$

In particular, $\varphi\left(L^{1}, L^{\infty}\right)$ coincides isometrically with the Orlicz space $L^{M}$ (see $[10,35$, 44]).

The Calderón-Lozanovskil construction is an exact interpolation method for positive linear or positive sublinear operators (see Berezhnoi [3], Shestakov [50], Maligranda [32]; $c f$. also [35, Theorem 15.13]). For arbitrary linear operators (not necessarily positive) on Banach ideal spaces with the Fatou property, this was proved by Ovchinnikov [43] (see also [10, 35, 42, 44] for the class of quasi-Banach ideal spaces). Some other properties of Calderón-Lozanovskil spaces were investigated in $[6,22,26]$.

The equivalence of two weights $u \approx v$ on $\Omega$ or $u(t) \approx v(t)$ on $\Omega$ will mean that there exists a constant $C>0$ such that $\frac{1}{C} u(t) \leq v(t) \leq C u(t)$ for all $t \in \Omega \mu$-a.e. Also $u \approx v$ simply means $u(t) \approx v(t)$ for all $t>0$.

Equality of two Banach spaces $X=Y$ means equality of $X$ and $Y$ as the sets and also equivalence of their norms. 


\section{Representation of Calderón-Lozanovskiĭ Spaces}

In the proof of the first representation theorem we will need the following lemma:

Lemma 1 Let $X, Y$ be two Banach ideal spaces with the Fatou property. If the norms $\|\cdot\|_{X}$ and $\|\cdot\|_{Y}$ are equivalent on $X \cap Y$, i.e., there exists a constant $C>0$ such that

$$
\frac{1}{C}\|x\|_{X} \leq\|x\|_{Y} \leq C\|x\|_{X} \quad \text { for all } x \in X \cap Y,
$$

then $X=Y$ with equivalent norms.

Proof Let $x \in X$ with the norm $\|x\|_{X} \leq 1$. In the Banach ideal space $X \cap Y$ with the natural norm $\|x\|_{X \cap Y}=\max \left\{\|x\|_{X},\|x\|_{Y}\right\}$ we take a unit function $u$, that is, the function $u \in X \cap Y$ such that $u(t)>0 \mu$-a.e. on $\Omega$. Then for the sequence of functions defined by

$$
x_{n}(t)=\min \{|x(t)|, n u(t)\}, \quad n \in N
$$

we have $x_{n} \in X \cap Y,\left\|x_{n}\right\|_{Y} \leq C, 0 \leq x_{n} \leq x_{n+1}$ and $\lim _{n \rightarrow \infty} x_{n}(t)=|x(t)| \mu$-a.e.

Using the Fatou property of $Y$ we obtain that $x \in Y$ and $\|x\|_{Y} \leq C$. Therefore, $X \subset Y$ and $\|x\|_{Y} \leq C\|x\|_{X}$ for all $x \in X$. Similarly we can prove the reverse imbedding, and Lemma 1 is proved.

Theorem 1 Let $\varphi \in \mathcal{U}$. Assume that $X_{0}, X_{1}$ and $X_{2}$ are Banach ideal spaces on the same $\sigma$-finite measure space $(\Omega, \mu)$, and suppose that all of the spaces have the Fatou property. If

$$
\varphi\left(X_{0}, X_{1}\right)=\varphi\left(X_{0}, X_{2}\right)
$$

and

$$
\lim _{n \rightarrow \infty} \inf _{s>0} \frac{\varphi(1, s n)}{\varphi(1, s)}=\infty
$$

then $X_{1}=X_{2}$.

Proof Assume that $X_{1} \neq X_{2}$. Then, by Lemma 1, we can find a sequence $x_{n} \in X_{1} \cap X_{2}$ such that $\left\|x_{n}\right\|_{X_{1}} \leq 1$ and $\left\|x_{n}\right\|_{X_{2}}>n$. Since $X_{2}$ has the Fatou property it follows that

$$
\sup _{\|y\|_{X_{2}^{\prime}}=1} \int_{\Omega}\left|x_{n}(t) y(t)\right| d \mu=\left\|x_{n}\right\|_{X_{2}^{\prime \prime}}=\left\|x_{n}\right\|_{X_{2}}>n,
$$

and so we can find a sequence $y_{n} \in X_{2}^{\prime},\left\|y_{n}\right\|_{X_{2}^{\prime}} \leq 1$ such that $\int_{\Omega}\left|x_{n}(t) y_{n}(t)\right| d \mu=n$.

Now, by the Lozanovskil factorization theorem (Theorem A), we can find sequences $0 \leq \xi_{n} \in X_{0}, 0 \leq \eta_{n} \in X_{0}^{\prime}$ such that

$$
\left\|\xi_{n}\right\|_{X_{0}}=1,\left\|\eta_{n}\right\|_{X_{0}^{\prime}}=1 \text { and } \frac{1}{n}\left|x_{n}(t) y_{n}(t)\right|=\xi_{n}(t) \eta_{n}(t) \quad \mu \text {-a.e. on } \Omega \text {. }
$$


We have $\varphi\left(\xi_{n}(t),\left|x_{n}(t)\right|\right) \in \varphi\left(X_{0}, X_{1}\right)$ with $\left\|\varphi\left(\xi_{n},\left|x_{n}\right|\right)\right\|_{\varphi\left(X_{0}, X_{1}\right)} \leq 1$, and

$$
\hat{\varphi}\left(\eta_{n}(t),\left|y_{n}(t)\right|\right) \in \hat{\varphi}\left(X_{0}^{\prime}, X_{2}^{\prime}\right)
$$

with $\left\|\hat{\varphi}\left(\eta_{n},\left|y_{n}\right|\right)\right\|_{\hat{\varphi}\left(X_{0}^{\prime}, X_{2}^{\prime}\right)} \leq 1$

Since $\hat{\varphi}\left(X_{0}^{\prime}, X_{2}^{\prime}\right)=\left[\varphi\left(X_{0}, X_{2}\right)\right]^{\prime}=\left[\varphi\left(X_{0}, X_{1}\right)\right]^{\prime}$ it follows that

$$
\begin{aligned}
A_{n} & :=\int_{\Omega} \varphi\left(\xi_{n}(t),\left|x_{n}(t)\right|\right) \hat{\varphi}\left(\eta_{n}(t),\left|y_{n}(t)\right|\right) d \mu \\
& \leq\left\|\varphi\left(\xi_{n},\left|x_{n}\right|\right)\right\|_{\varphi\left(X_{0}, X_{1}\right)}\left\|\hat{\varphi}\left(\eta_{n},\left|y_{n}\right|\right)\right\|_{\varphi\left(X_{0}, X_{1}\right)^{\prime}} \\
& \leq\left\|\hat{\varphi}\left(\eta_{n},\left|y_{n}\right|\right)\right\|_{\varphi\left(X_{0}, X_{1}\right)^{\prime}} \\
& \leq C\left\|\hat{\varphi}\left(\eta_{n},\left|y_{n}\right|\right)\right\|_{\hat{\varphi}\left(X_{0}^{\prime}, X_{2}^{\prime}\right)} \leq C
\end{aligned}
$$

which gives that $\sup _{n \in \mathrm{N}} A_{n}<\infty$.

On the other hand, by an estimate in $(1)$, we have $\hat{\varphi}(1, u) \varphi\left(1, \frac{1}{u}\right) \geq 1$ and

$$
\begin{aligned}
A_{n} & =\int_{\Omega} \xi_{n}(t) \eta_{n}(t) \varphi\left(1, \frac{\left|x_{n}(t)\right|}{\xi_{n}(t)}\right) \hat{\varphi}\left(1, \frac{\left|y_{n}(t)\right|}{\eta_{n}(t)}\right) d \mu \\
& \geq \int_{\Omega} \xi_{n}(t) \eta_{n}(t) \varphi\left(1, \frac{\left|x_{n}(t)\right|}{\xi_{n}(t)}\right) \frac{1}{\varphi\left(1, \frac{\eta_{n}(t)}{\mid y_{n}(t)}\right)} d \mu \\
& =\int_{\Omega} \xi_{n}(t) \eta_{n}(t) \frac{\varphi\left(1, \frac{n \eta_{n}(t)}{\left|y_{n}(t)\right|}\right)}{\varphi\left(1, \frac{\eta_{n}(t)}{\left|y_{n}(t)\right|}\right)} d \mu \\
& \geq \inf _{s>0} \frac{\varphi(1, n s)}{\varphi(1, s)} \int_{\Omega} \xi_{n}(t) \eta_{n}(t) d \mu=\inf _{s>0} \frac{\varphi(1, n s)}{\varphi(1, s)},
\end{aligned}
$$

that is,

$$
\sup _{n \in \mathbf{N}} A_{n} \geq \sup _{n \in \mathbf{N}} \inf _{s>0} \frac{\varphi(1, n s)}{\varphi(1, s)} \geq \lim _{n \rightarrow \infty} \inf _{s>0} \frac{\varphi(1, s n)}{\varphi(1, s)}=\infty
$$

which gives a contradiction. Therefore $X_{1}=X_{2}$ and the norms are equivalent.

Theorem 1, used in the case of power function $\varphi_{\theta}(s, t)=s^{1-\theta} t^{\theta}$ with $0<\theta<1$, gives the following corollary, which was proved differently by Cwikel and Nilsson [14, Theorem 3.5].

Corollary 1 Let $0<\theta<1$. If $X_{0}^{1-\theta} X_{1}^{\theta}=X_{0}^{1-\theta} X_{2}^{\theta}$ for Banach ideal spaces $X_{0}, X_{1}$ and $X_{2}$ on $(\Omega, \mu)$ with the Fatou property, then $X_{1}=X_{2}$.

Remark 1 For concrete spaces, the assumption (3) on $\varphi$ can be weakened, as we will prove in Theorem 4. Let $\varphi \in \mathcal{U}$ and $\lim _{t \rightarrow \infty} \varphi(t, 1)=\infty$. Assume that the measure space $(\Omega, \mu)$ is nonatomic. If

$$
\varphi\left(L_{u}^{1}, L^{\infty}\right)=\varphi\left(L_{w}^{1}, L^{\infty}\right)
$$


with equivalent norms for some weight functions $u$, w on $\Omega$, then $u(t) \approx w(t)$ on $\Omega$.

Using Theorem 1 we can prove the following uniqueness theorem for two couples $\bar{X}=\left(X_{0}, X_{1}\right)$ and $\bar{Y}=\left(Y_{0}, Y_{1}\right)$ of Banach ideal spaces with the Fatou property.

Theorem 2 Let $\bar{X}=\left(X_{0}, X_{1}\right)$ and $\bar{Y}=\left(Y_{0}, Y_{1}\right)$ be two couples of Banach ideal spaces on the same measure space $(\Omega, \mu)$ with all spaces having the Fatou property. Suppose that for $\varphi_{0}, \varphi_{1} \in \mathcal{U}$ we can find $\varphi \in \mathcal{U}$ such that either

$$
\varphi\left(\varphi_{0}(s, 1), 1\right)=\varphi_{1}(s, 1) \text { for all } s>0 \text { or } \varphi\left(1, \varphi_{0}(1, t)\right)=\varphi_{1}(1, t) \text { for all } t>0 .
$$

Assume also that $\varphi$ satisfies (3) and either $\varphi_{0}$ or $\varphi_{1}$ satisfies

$$
\lim _{n \rightarrow \infty} \inf _{s>0} \frac{\varphi_{i}(s n, 1)}{\varphi_{i}(s, 1)}=\infty .
$$

If $\varphi_{0}\left(X_{0}, X_{1}\right)=\varphi_{0}\left(Y_{0}, Y_{1}\right)$ and $\varphi_{1}\left(X_{0}, X_{1}\right)=\varphi_{1}\left(Y_{0}, Y_{1}\right)$, then $X_{0}=Y_{0}$ and $X_{1}=Y_{1}$.

Proof By the reiteration formulas (see [35, pp. 180-181]) it yields that

$$
\varphi\left(\varphi_{0}\left(X_{0}, X_{1}\right), X_{1}\right)=\varphi_{1}\left(X_{0}, X_{1}\right) \text { and } \varphi\left(\varphi_{0}\left(Y_{0}, Y_{1}\right), Y_{1}\right)=\varphi_{1}\left(Y_{0}, Y_{1}\right) .
$$

From the equalities in the assumption we obtain that

$$
\varphi\left(X, X_{1}\right)=\varphi\left(X, Y_{1}\right) \text { with } X=\varphi_{0}\left(X_{0}, X_{1}\right) .
$$

Using Theorem 1 we obtain that $X_{1}=Y_{1}$ with equivalent norms. Now, if $\varphi_{i}$ satisfies (4) for $i=0$ or $i=1$, then from the first or the second equality in the assumption and from the just proved equality $X_{1}=Y_{1}$ we have

$$
\varphi_{i}\left(X_{0}, X_{1}\right)=\varphi_{i}\left(Y_{0}, X_{1}\right), \quad i=0,1,
$$

or

$$
\tilde{\varphi}_{i}\left(X_{1}, X_{0}\right)=\tilde{\varphi}_{i}\left(X_{1}, Y_{0}\right), \quad i=0,1,
$$

where $\tilde{\varphi}_{i}(s, t)=\varphi_{i}(t, s)$. Since the condition (4) for $\varphi_{i}$ means the condition (3) for $\tilde{\varphi}_{i}$ we obtain from Theorem 1 that $X_{0}=Y_{0}$ with equivalent norms, and the proof is complete.

As a corollary, we obtain the result proved by Cwikel and Nilsson [14, Theorem 3.1] for the power functions $\varphi_{\theta_{0}}$ and $\varphi_{\theta_{1}}$.

Corollary 2 If $X_{0}^{1-\theta_{0}} X_{1}^{\theta_{0}}=Y_{0}^{1-\theta_{0}} Y_{1}^{\theta_{0}}$ and $X_{0}^{1-\theta_{1}} X_{2}^{\theta_{1}}=Y_{0}^{1-\theta_{1}} Y_{1}^{\theta_{1}}$ for some $\theta_{0}, \theta_{1} \in$ $[0,1]$ with $\theta_{0} \neq \theta_{1}$ and for Banach ideal spaces $X_{0}, X_{1}, Y_{0}, Y_{1}$ on $(\Omega, \mu)$, all with the Fatou property, then $X_{0}=Y_{0}$ and $X_{1}=Y_{1}$.

Theorem 3 Let $X, Y$ be two Banach ideal spaces on $\Omega$ and $u, v$ two weights on $\Omega$. Then for $0 \leq \theta \leq 1$ we have equality

$$
X^{1-\theta} Y^{\theta}=\left(X_{u}\right)^{1-\theta}\left(Y_{v}\right)^{\theta}
$$

if and only if $u(t)^{1-\theta} v(t)^{\theta} \approx C$ on $\Omega$. 
Proof Let $a \leq u(t)^{1-\theta} v(t)^{\theta} \leq b$ for some $a, b>0$ and all $t \in \Omega \mu$-a.e.

If $x \in X^{1-\theta} Y^{\theta}$ with norm $<1$, then

$$
|x| \leq\left|x_{0}\right|^{1-\theta}\left|x_{1}\right|^{\theta} \text { with }\left\|x_{0}\right\|_{X} \leq 1 \text { and }\left\|x_{1}\right\|_{Y} \leq 1,
$$

which we can rewrite as

$$
|x| \leq b\left|\frac{x_{0}}{u}\right|^{1-\theta}\left|\frac{x_{1}}{v}\right|^{\theta}=b\left|x_{0}^{\prime}\right|^{1-\theta}\left|x_{1}^{\prime}\right|^{\theta}
$$

with $\left\|x_{0}^{\prime}\right\|_{X_{u}}=\left\|x_{0}\right\|_{X} \leq 1$ and $\left\|x_{1}^{\prime}\right\|_{Y_{v}}=\left\|x_{1}\right\|_{Y} \leq 1$. This means that

$$
x \in\left(X_{u}\right)^{1-\theta}\left(Y_{v}\right)^{\theta}
$$

with norm $\leq b$.

Conversely, if $x \in\left(X_{u}\right)^{1-\theta}\left(Y_{v}\right)^{\theta}$ with norm $<1$, then

$$
|x| \leq\left|x_{0}\right|^{1-\theta}\left|x_{1}\right|^{\theta} \text { with }\left\|x_{0}\right\|_{X_{u}} \leq 1 \text { and }\left\|x_{1}\right\|_{Y_{v}} \leq 1,
$$

which gives

$$
|x| \leq \frac{1}{a}\left|x_{0} u\right|^{1-\theta}\left|x_{1} v\right|^{\theta}=\frac{1}{a}\left|x_{0}^{\prime}\right|^{1-\theta}\left|x_{1}^{\prime}\right|^{\theta}
$$

with $\left\|x_{0}^{\prime}\right\|_{X}=\left\|x_{0}\right\|_{X_{u}} \leq 1$ and $\left\|x_{1}^{\prime}\right\|_{Y}=\left\|x_{1}\right\|_{Y_{v}} \leq 1$, that is, $x \in X^{1-\theta} Y^{\theta}$ with norm $\leq \frac{1}{a}$.

To prove the reverse implication assume that $X^{1-\theta} Y^{\theta}=\left(X_{u}\right)^{1-\theta}\left(Y_{v}\right)^{\theta}$. Then, by the duality theorem,

$$
\left(X^{\prime}\right)^{1-\theta}\left(Y^{\prime}\right)^{\theta}=\left(X_{1 / u}^{\prime}\right)^{1-\theta}\left(Y_{1 / v}^{\prime}\right)^{\theta}
$$

and for any non-negative functions $x_{0} \in X, x_{1} \in Y, y_{0} \in X^{\prime}, y_{1} \in Y^{\prime}$ from the unit balls, we have

$$
\begin{aligned}
\int_{\Omega} x_{0}(t)^{1-\theta} x_{1}(t)^{\theta} y_{0}(t)^{1-\theta} y_{1}(t)^{\theta} u(t)^{1-\theta} v(t)^{\theta} d \mu \\
\leq\left\|x_{0}^{1-\theta} x_{1}^{\theta}\right\|_{X^{1-\theta} Y^{\theta}}\left\|\left(y_{0} u\right)^{1-\theta}\left(y_{1} v\right)^{\theta}\right\|_{\left(X^{1-\theta} Y^{\theta}\right)^{\prime}} \\
=\left\|x_{0}^{1-\theta} x_{1}^{\theta}\right\|_{X^{1-\theta} Y^{\theta}}\left\|\left(y_{0} u\right)^{1-\theta}\left(y_{1} v\right)^{\theta}\right\|_{\left(X^{\prime}\right)^{1-\theta}\left(Y^{\prime}\right)^{\theta}} \\
\leq C\left\|x_{0}^{1-\theta} x_{1}^{\theta}\right\|_{X^{1-\theta} Y^{\theta}}\left\|\left(y_{0} u\right)^{1-\theta}\left(y_{1} v\right)^{\theta}\right\|_{\left(X^{\prime}{ }_{1 / u}\right)^{1-\theta}\left(Y^{\prime}{ }_{1 / v}\right)^{\theta}} \\
\leq C\left\|x_{0}\right\|_{X}^{1-\theta}\left\|x_{1}\right\|_{Y}^{\theta}\left\|y_{0} u\right\|_{X^{\prime}{ }_{1 / u}}^{1-\theta}\left\|y_{1} v\right\|_{Y^{\prime}{ }_{1 / v}}^{\theta} \leq C .
\end{aligned}
$$

By the factorization theorem (Theorem A), for any $0 \leq z \in L^{1}(\mu)$ we can find nonnegative $x_{0} \in X, y_{0} \in X^{\prime}$ and $x_{1} \in Y, y_{1} \in Y^{\prime}$ such that $x_{0} y_{0}=z$ and $x_{1} y_{1}=z$. Then

$$
\int_{\Omega} z(t) u(t)^{1-\theta} v(t)^{\theta} d \mu=\int_{0}^{\infty} x_{0}(t)^{1-\theta} x_{1}(t)^{\theta} y_{0}(t)^{1-\theta} y_{1}(t)^{\theta} u(t)^{1-\theta} v(t)^{\theta} d \mu \leq C
$$

from which we obtain that $u(t)^{1-\theta} v(t)^{\theta} \in L^{\infty}(\mu)$, or equivalently $u(t)^{1-\theta} v(t)^{\theta} \leq C$ for all $t \in \Omega \mu$-a.e., since the following implication is true: 
If $0 \leq w \notin L^{\infty}(\mu)$, then we can find $0 \leq z \in L^{1}(\mu)$ for which $\int_{\Omega} z(t) w(t) d \mu=\infty$.

Now, for the spaces $X_{0}=X_{u}$ and $X_{1}=Y_{v}$ we have by the formula in the assumption that

$$
\left(\left(X_{0}\right)_{1 / u}\right)^{1-\theta}\left(\left(X_{1}\right)_{1 / v}\right)^{\theta}=X^{1-\theta} Y^{\theta}=\left(X_{u}\right)^{1-\theta}\left(Y_{v}\right)^{\theta}=X_{0}^{1-\theta} X_{1}^{\theta}
$$

Therefore the equality $\left(\left(X_{0}\right)_{1 / u}\right)^{1-\theta}\left(\left(X_{1}\right)_{1 / v}\right)^{\theta}=X_{0}^{1-\theta} X_{1}^{\theta}$ holds, from which together with the proof as above we obtain that $\left(\frac{1}{u}\right)^{1-\theta}\left(\frac{1}{v}\right)^{\theta} \in L^{\infty}(\mu)$ or, equivalently, $u(t)^{1-\theta} v(t)^{\theta} \geq c>0$ for all $t \in \Omega \mu$-a.e. Thus $u(t)^{1-\theta} v(t)^{\theta}$ is equivalent to a constant function on $\Omega$.

Corollary 3 Let $X, Y$ be two Banach ideal spaces on $\Omega$ and $u, v$ two weights on $\Omega$. If we have equalities

$$
X^{1-\theta_{0}} Y^{\theta_{0}}=\left(X_{u}\right)^{1-\theta_{0}}\left(Y_{v}\right)^{\theta_{0}} \text { and } X^{1-\theta_{1}} Y^{\theta_{1}}=\left(X_{u}\right)^{1-\theta_{1}}\left(Y_{v}\right)^{\theta_{1}}
$$

for some $\theta_{0}, \theta_{1} \in[0,1]$ with $\theta_{0} \neq \theta_{1}$, then $u(t) \approx v(t) \approx C$ on $\Omega$.

Proof From Theorem 3, used twice, we have that $u^{1-\theta_{0}} v^{\theta_{0}}=u\left(\frac{v}{u}\right)^{\theta_{0}} \approx C_{0}$ on $\Omega$ and $u^{1-\theta_{1}} v^{\theta_{1}}=u\left(\frac{v}{u}\right)^{\theta_{1}} \approx C_{1}$ on $\Omega$. Therefore, $u(t) \approx v(t) \approx C$ on $\Omega$.

The next theorem on the representation or the inverse interpolation problem will have only weighted $L^{1}$ and $L^{\infty}$ spaces but then we can change the spaces on both places. We again need a lemma.

Lemma 2 If $\varphi(t, 1)$ is a strictly increasing function and for some $x \in X$ and some measurable set $A$ we have $\left\|x \chi_{A}\right\|_{X}=1$, then $\left\|\varphi\left(|x|, v^{-1}\right) \chi_{A}\right\|_{\varphi\left(X, L_{v}^{\infty}\right)}=1$.

Proof Clearly $\left\|\varphi\left(|x|, v^{-1}\right) \chi_{A}\right\|_{\varphi\left(X, L_{v}^{\infty}\right)} \leq 1$. Assume therefore that it is strictly less than 1 . Then, for some $\varepsilon>0$,

$$
\varphi\left(|x|, v^{-1}\right) \chi_{A} \leq(1-\varepsilon) \varphi\left(\left|x_{0}\right|,\left|x_{1}\right|\right) \chi_{A}
$$

with $\left\|x_{0}\right\|_{X} \leq 1$ and $\left\|x_{1}\right\|_{L_{v}^{\infty}} \leq 1$. Hence

$$
\varphi\left(|x|, v^{-1}\right) \chi_{A} \leq(1-\varepsilon) \varphi\left(\left|x_{0}\right|, v^{-1}\right) \chi_{A} \leq \varphi\left((1-\varepsilon)\left|x_{0}\right|, v^{-1}\right) \chi_{A} .
$$

Since $\varphi(t, 1)$ is strictly increasing it follows that $|x(t)| \chi_{A} \leq(1-\varepsilon)\left|x_{0}\right| \chi_{A}$ and so $\left\|x \chi_{A}\right\|_{X} \leq(1-\varepsilon)\left\|x_{0} \chi_{A}\right\|_{X} \leq(1-\varepsilon)<1$, which is a contradiction.

For a function $\varphi \in \mathcal{U}$ consider a submultiplicative and quasi-concave function $\rho_{\varphi}$ on $(0, \infty)$ defined by

$$
\rho_{\varphi}(a)=\limsup _{t \rightarrow \infty} \frac{\varphi(a t, 1)}{\varphi(t, 1)}, \quad a>0 .
$$


By the well-known theorem on submultiplicative functions on $(0, \infty)$ we can find numbers $0 \leq \alpha \leq \beta \leq 1$, called also the indices of $\varphi$, such that

$$
\rho_{\varphi}(a) \geq \max \left(a^{\alpha}, a^{\beta}\right)
$$

Moreover, for any $\varepsilon>0$ we have $\rho_{\varphi}(a) \leq a^{\alpha-\varepsilon}$ for $a>0$ sufficiently close to zero, $\rho_{\varphi}(a) \leq a^{\beta+\varepsilon}$ for $a$ sufficiently large, and

$$
\alpha=\alpha_{\varphi}=\lim _{a \rightarrow 0^{+}} \frac{\ln \rho_{\varphi}(a)}{\ln a}, \beta=\beta_{\varphi}=\lim _{a \rightarrow \infty} \frac{\ln \rho_{\varphi}(a)}{\ln a}
$$

(see, e.g., [25], Theorem 1.3 or [35], Theorem 11.3).

Theorem 4 Let $\varphi \in \mathcal{U}$ and $\varphi(t, 1)$ be a strictly increasing function. Assume that the measure space $(\Omega, \mu)$ is nonatomic. If

$$
\varphi\left(L_{u}^{1}, L_{v}^{\infty}\right)=\varphi\left(L_{w}^{1}, L^{\infty}\right)
$$

for some weight functions $u, v, w$, then there exists $\theta \in[0,1]$ such that

$$
w(t)^{\theta} \approx u(t)^{\theta} v(t)^{1-\theta} \quad \text { on } \Omega .
$$

More precisely, if $v$ is equivalent to a constant function, then we can take $\theta=0$ and if $v$ is not equivalent to a constant function on $\Omega$, then the function $\varphi$ has the same indices $\alpha_{\varphi}=\beta_{\varphi}$ and we can take $\theta=\alpha=\alpha_{\varphi}$.

Proof If $v$ is equivalent to a constant function on $\Omega$, i.e.,

$$
c=\sup _{t \in \Omega} \frac{1}{v(t)} \sup _{t \in \Omega} v(t)<\infty
$$

then we can take $\theta=0$.

Assume therefore that $\sup _{t \in \Omega} \frac{1}{v(t)} \sup _{t \in \Omega} v(t)=\infty$. For any $k \in \mathbf{N}$ define sets

$$
\begin{gathered}
U_{k}=\left\{t \in \Omega: 2^{-k-1}<u(t) \leq 2^{-k}\right\}, V_{k}=\left\{t \in \Omega: 2^{-k-1}<v(t) \leq 2^{-k}\right\}, \\
W_{k}=\left\{t \in \Omega: 2^{-k-1}<w(t) \leq 2^{-k}\right\}, P=\left\{(i, j, k) \in \mathbf{N}^{3}: U_{i} \cap V_{j} \cap W_{k} \neq \varnothing\right\} .
\end{gathered}
$$

Note that $\bigcup_{(i, j, k) \in P} U_{i} \cap V_{j} \cap W_{k}=\Omega$. If $0<\mu(A)<\infty$, then $\left\|\frac{1}{u \mu(A)} \chi_{A}\right\|_{L_{u}^{1}}=1$ and by Lemma 2 we have the equality

$$
\left\|\varphi\left(\frac{1}{u \mu(A)}, \frac{1}{v}\right) \chi_{A}\right\|_{\varphi\left(L_{u}^{1}, L_{v}^{\infty}\right)}=1 .
$$

If $A \subset U_{i} \cap V_{j} \cap W_{k}$, then

$$
\left\|\varphi\left(\frac{2^{i}}{\mu(A)}, 2^{j}\right) \chi_{A}\right\|_{\varphi\left(L_{u}^{1}, L_{v}^{\infty}\right)} \leq\left\|\varphi\left(\frac{1}{u \mu(A)}, \frac{1}{v}\right) \chi_{A}\right\|_{\varphi\left(L_{u}^{1}, L_{v}^{\infty}\right)}=1
$$


and, by the assumption that the norms are equivalent,

$$
\left\|\varphi\left(\frac{2^{i}}{\mu(A)}, 2^{j}\right) \chi_{A}\right\|_{\varphi\left(L_{w}^{1}, L^{\infty}\right)} \leq C
$$

and so

$$
\varphi\left(\frac{2^{i}}{\mu(A)}, 2^{j}\right) \chi_{A}(t) \leq C \varphi\left(\frac{|x(t)|}{w(t)}, 1\right) \chi_{A}(t)
$$

with $x \in L^{1},\|x\|_{L^{1}} \leq 1$ or

$$
\varphi\left(\frac{2^{i}}{\mu(A)}, 2^{j}\right) \chi_{A}(t) \leq 2 C \varphi\left(|x(t)| 2^{k}, 1\right) \chi_{A}(t) .
$$

Take $d=\operatorname{ess~inf}_{t \in A}|x(t)|$. If $d>0$, then

$$
\varphi\left(\frac{2^{i}}{\mu(A)}, 2^{j}\right) \chi_{A}(t) \leq 2 C \varphi\left(d 2^{k}, 1\right) \chi_{A}(t)
$$

and $d \chi_{A}(t) \leq|x(t)| \chi_{A}(t)$ gives $\left\|d \chi_{A}\right\|_{L^{1}} \leq\left\|x \chi_{A}\right\|_{L^{1}} \leq 1$ or $d \leq \frac{1}{\mu(A)}$. Thus

$$
\varphi\left(\frac{2^{i}}{\mu(A)}, 2^{j}\right) \chi_{A}(t) \leq 2 C \varphi\left(\frac{1}{\mu(A)} 2^{k}, 1\right) \chi_{A}(t) .
$$

If $d=0$, then $\lim _{t \rightarrow 0^{+}} \varphi(t, 1)>0$, and the above estimate also holds.

Similarly, for $A \subset U_{i} \cap V_{j} \cap W_{k}$ with $0<\mu(A)<\infty$ we have the estimate

$$
\varphi\left(\frac{1}{\mu(A)} 2^{k}, 1\right) \chi_{A}(t) \leq 2 C \varphi\left(\frac{2^{i}}{\mu(A)}, 2^{j}\right) \chi_{A}(t) .
$$

From the above estimates we have the inequalities

$$
\frac{1}{2 C} \varphi\left(\frac{2^{k}}{\mu(A)}, 1\right) \leq 2^{j} \varphi\left(2^{i-j-k} \frac{2^{k}}{\mu(A)}, 1\right) \leq 2 C \varphi\left(\frac{2^{k}}{\mu(A)}, 1\right),
$$

and by taking $\mu(A) \rightarrow 0^{+}$(we can do this since measure is nonatomic) we have

$$
2^{j} \rho_{\varphi}\left(2^{i-j-k}\right) \leq 2 C \text { and } 2^{-j} \rho_{\varphi}\left(2^{j+k-i}\right) \leq 2 C .
$$

Let $Q=\{p=i-j-k:(i, j, k) \in P\}$. If $\sup \{|p|: p \in Q\}<\infty$, then $2^{j} \leq 2 C$, $2^{-j} \leq 2 C$ and the weight $v(t)$ is equivalent to a constant function, which cannot happen. Thus we must have

$$
\sup \{|p|: p \in Q\}=\infty .
$$

Since

$$
\rho_{\varphi}\left(2^{i-j-k}\right) \rho_{\varphi}\left(2^{j+k-i}\right) \leq 4 C^{2}
$$


it follows that

$$
\alpha=\beta, \rho_{\varphi}(a) \geq a^{\alpha} \text { for all } a>0 \text {, and } \rho_{\varphi}\left(2^{p}\right) \rho_{\varphi}\left(2^{-p}\right) \leq 4 C^{2} \text { for all } p \in Q .
$$

If $\sup \{p: p \in Q\}=\infty$ and $\lim \sup _{p \rightarrow \infty, p \in Q} \frac{\rho_{\varphi}\left(2^{p}\right)}{2^{p \alpha}}=\infty$, then

$$
4 C^{2} \geq \limsup _{p \rightarrow \infty, p \in Q} \rho_{\varphi}\left(2^{p}\right) \rho_{\varphi}\left(2^{-p}\right) \geq \limsup _{p \rightarrow \infty, p \in Q} \rho_{\varphi}\left(2^{p}\right)\left(2^{-\alpha p}\right)=\infty .
$$

If $\inf \{p: p \in Q\}=-\infty$ and $\lim \sup _{p \rightarrow-\infty, p \in Q} \frac{\rho_{\varphi}\left(2^{p}\right)}{2^{p \alpha}}=\infty$, then

$$
4 C^{2} \geq \limsup _{p \rightarrow \infty, p \in Q} \rho_{\varphi}\left(2^{p}\right) \rho_{\varphi}\left(2^{-p}\right) \geq \limsup _{p \rightarrow \infty, p \in Q} \rho_{\varphi}\left(2^{p}\right)\left(2^{-\alpha p}\right)=\infty .
$$

This means that $2^{j} 2^{(i-j-k) \alpha} \approx 1$ for all $(i, j, k) \in P$. Therefore on all sets $U_{i} \cap V_{j} \cap W_{k}$ we have $\left(\frac{1}{u}\right)^{\alpha}\left(\frac{1}{v}\right)^{1-\alpha} w^{\alpha} \approx 1$ or $w^{\alpha} \approx u^{\alpha} v^{1-\alpha}$, and since the sum of these sets is $\Omega$, the proof is complete.

In equality (5) we can have four weights, but before we formulate it we prove the following lemma:

Lemma 3 The equality $\varphi\left(L_{u_{0}}^{1}, L_{u_{1}}^{\infty}\right)=\varphi\left(L_{v_{0}}^{1}, L_{v_{1}}^{\infty}\right)$ holds if and only if the equality $\varphi\left(L_{u_{0} w}^{1}, L_{u_{1} w}^{\infty}\right)=\varphi\left(L_{v_{0} w}^{1}, L_{v_{1} w}^{\infty}\right)$ is true.

Proof It is enough to show that

$$
\varphi\left(L_{u_{0}}^{1}, L_{u_{1}}^{\infty}\right) \subset \varphi\left(L_{v_{0}}^{1}, L_{v_{1}}^{\infty}\right) \text { implies } \varphi\left(L_{u_{0} w}^{1}, L_{u_{1} w}^{\infty}\right) \subset \varphi\left(L_{v_{0} w}^{1}, L_{v_{1} w}^{\infty}\right)
$$

with the same norms of embeddings. In fact, if $x \in \varphi\left(L_{u_{0} w}^{1}, L_{u_{1} w}^{\infty}\right)$ with the norm $<1$, then

$$
|x| \leq \varphi\left(\left|x_{0}\right|,\left|x_{1}\right|\right) \text { with }\left\|x_{0}\right\|_{L_{u_{0} w}^{1}} \leq 1 \text { and }\left\|x_{1}\right\|_{L_{u_{1} w}^{\infty}} \leq 1,
$$

and so

$$
|x| w \leq \varphi\left(\left|x_{0}\right| w,\left|x_{1}\right| w\right)=\varphi\left(y_{0}, y_{1}\right)
$$

with

$$
\left\|y_{0}\right\|_{L_{u_{0}}^{1}}=\left\|x_{0} w\right\|_{L_{u_{0}}^{1}}=\left\|x_{0}\right\|_{L_{u_{0} w}^{1}} \leq 1 \text { and }\left\|y_{1}\right\|_{L_{u_{1}}^{\infty}}=\left\|x_{1} w\right\|_{L_{u_{1}}^{\infty}}=\left\|x_{1}\right\|_{L_{u_{1} w}^{\infty}} \leq 1 \text {. }
$$

Thus $x w \in \varphi\left(L_{u_{0}}^{1}, L_{u_{1}}^{\infty}\right)$ and, by the embedding assumption, $x w \in \varphi\left(L_{v_{0}}^{1}, L_{v_{1}}^{\infty}\right)$, that is,

$$
|x| w \leq C \varphi\left(\left|z_{0}\right|,\left|z_{1}\right|\right) \text { with }\left\|z_{0}\right\|_{L_{v_{0}}^{1}} \leq 1 \text { and }\left\|z_{1}\right\|_{L_{v_{1}}^{\infty}} \leq 1
$$

or, equivalently,

$$
|x| \leq C \varphi\left(\frac{\left|z_{0}\right|}{w}, \frac{\left|z_{1}\right|}{w}\right)=C \varphi\left(x_{0}^{\prime}, x_{1}^{\prime}\right)
$$


with

$$
\left\|x_{0}^{\prime}\right\|_{L_{v_{0} w}^{1}}=\left\|\frac{z_{0}}{w}\right\|_{L_{v_{0} w}^{1}}=\left\|z_{0}\right\|_{L_{v_{0}}^{1}} \leq 1 \text { and }\left\|x_{1}^{\prime}\right\|_{L_{v_{1} w}^{\infty}}=\left\|\frac{z_{1}}{w}\right\|_{L_{v_{1} w}^{\infty}}=\left\|z_{1}\right\|_{L_{v_{1}}^{\infty} \leq 1,} \leq 1,
$$

we obtain that $x \in \varphi\left(L_{v_{0} w}^{1}, L^{\infty}\right)_{v_{1} w}$ with the norm $\leq C$.

Directly from Theorem 4 and Lemma 3 we obtain the following result:

Corollary 4 Let $\varphi$ and the measure space $(\Omega, \mu)$ be the same as in Theorem 4 . If

$$
\varphi\left(L_{u_{0}}^{1}, L_{u_{1}}^{\infty}\right)=\varphi\left(L_{v_{0}}^{1}, L_{v_{1}}^{\infty}\right)
$$

for some weight functions $u_{0}, u_{1}, v_{0}, v_{1}$ on $\Omega$, then there exists $\theta \in[0,1]$ such that

$$
u_{0}(t)^{\theta} u_{1}(t)^{1-\theta} \approx v_{0}(t)^{\theta} v_{1}(t)^{1-\theta} \text { on } \Omega
$$

Remark 2 Note that

$$
\varphi\left(L_{u}^{1}, L_{v}^{\infty}\right)=L_{v}^{M}\left(\frac{u}{v} d t\right)
$$

where function $M$ is defined by $M(\varphi(s, 1))=s$ and the last space is a weighted Orlicz space generated by the norm

$$
\|x\|_{L_{v}^{M}\left(\frac{u}{v} d t\right)}=\inf \left\{\lambda>0: \int_{\Omega} M(v(t)|x(t)| / \lambda) \frac{u(t)}{v(t)} d t \leq 1\right\}
$$

Similarly, $\varphi\left(L_{w}^{1}, L^{\infty}\right)=L^{M}(w d t)$. In the case when $v=1$ and $M \in \Delta_{2}$ globally, that is, $M(2 u) \leq C M(u)$ for all $u>0$, it is known that $L^{M}(u d t)=L^{M}(w d t)$ if and only if $u \approx w$ on $(0, \infty)$ or on a measurable subset $\Omega$ of $R^{n}$ of a positive measure (see [24]). In the case when $v$ is not equivalent to a constant, then the technique from [24] does not work. On the other hand, if we look for these spaces as special cases of the Musielak-Orlicz spaces generated by the functions $\mathcal{M}(a, t)=M(v(t) a) \frac{u(t)}{v(t)}$ and $\mathcal{N}(a, t)=M(a) w(t)$ and use the criterion for the equality $L^{\mathcal{N}}=L^{\mathcal{N}}$ with equivalent norms (see [40]), then these general conditions seem to be not helpful in proving the corresponding equivalence of the weights as in Corollary 4.

We give an example showing that for a certain non-power function $\varphi \in \mathcal{U}$ and some weights $u, v$ we can have equality $\varphi\left(L_{u}^{1}, L_{v}^{\infty}\right)=\varphi\left(L^{1}, L^{\infty}\right)$ with equivalent norms.

Example 1 Consider the concave function on $(0, \infty)$ defined by $\psi(t)=t^{1 / 2} \ln ^{1 / 2}(1+$ $t$ ) and let $\varphi(s, t)=t \psi(s / t)=s^{1 / 2} t^{1 / 2} \ln ^{1 / 2}\left(1+\frac{s}{t}\right)$. Then $\rho_{\varphi}(a)=a^{1 / 2}$. We will show that there exists a weight $u$ on $\Omega=I=(0,1)$ such that

$$
\varphi\left(L_{1 / u}^{1}(I), L_{u}^{\infty}(I)\right)=\varphi\left(L^{1}(I), L^{\infty}(I)\right)
$$

with equivalent norms. Assume that the weight $u$ satisfies $u(t) \geq 1$ a.e. on $I$ and $\int_{0}^{1} u(t) d t \leq 2$. 
Observe that for $a, b \geq 0$ we have the inequality

$$
a \ln \left(1+a b^{2}\right) \leq 2(a+b) \ln (1+a+b) .
$$

In fact, if $0 \leq a \leq 1$, then

$$
\begin{aligned}
a \ln \left(1+a b^{2}\right) & \leq a \ln \left(1+b^{2}\right) \leq a \ln (1+b)^{2} \\
& =2 a \ln (1+b) \leq 2(a+b) \ln (1+a+b),
\end{aligned}
$$

and if $a \geq 1$, then

$$
\begin{aligned}
a \ln \left(1+a b^{2}\right) & \leq a \ln \left(a+a b^{2}\right) \leq a \ln (1+a+b)^{2} \\
& =2 a \ln (1+a+b) \leq 2(a+b) \ln (1+a+b) .
\end{aligned}
$$

We show first the imbedding $\varphi\left(L_{1 / u}^{1}, L_{u}^{\infty}\right) \subset \varphi\left(L^{1}, L^{\infty}\right)$. If $x \in \varphi\left(L_{1 / u}^{1}, L_{u}^{\infty}\right)$ and the norm is $<1$, then

$$
|x| \leq \varphi\left(\left|x_{0}\right| u, \frac{1}{u}\right) \text { with }\left\|x_{0}\right\|_{L^{1}} \leq 1
$$

and, by (7),

$$
\begin{aligned}
|x| \leq \varphi\left(\left|x_{0}\right| u, \frac{1}{u}\right) & =\left|x_{0}\right|^{1 / 2} \ln ^{1 / 2}\left(1+\left|x_{0}\right| u^{2}\right) \leq \sqrt{2}\left(\left|x_{0}\right|+u\right)^{1 / 2} \ln ^{1 / 2}\left(1+\left|x_{0}\right|+u\right) \\
& =\sqrt{2} \varphi\left(\left|x_{0}\right|+u, 1\right) \leq 3 \sqrt{2} \varphi\left(\frac{\left|x_{0}\right|+u}{3}, 1\right) .
\end{aligned}
$$

This means that $x \in \varphi\left(L^{1}, L^{\infty}\right)$ with norm $\leq 3 \sqrt{2}$. Therefore we have a continuous imbedding

$$
\varphi\left(L_{1 / u}^{1}, L_{u}^{\infty}\right) \stackrel{3 \sqrt{2}}{\hookrightarrow} \varphi\left(L^{1}, L^{\infty}\right) .
$$

Secondly, we prove the reverse imbedding $\varphi\left(L^{1}, L^{\infty}\right) \subset \varphi\left(L_{1 / u}^{1}, L_{u}^{\infty}\right)$. Let $x \in$ $\varphi\left(L^{1}, L^{\infty}\right)$ with norm $<1$, that is,

$$
|x| \leq \varphi\left(\left|x_{0}\right|, 1\right) \text { and }\left\|x_{0}\right\|_{L^{1}} \leq 1
$$

Then, since the weight $\mathrm{u}$ satisfies $u(t) \geq 1$ a.e on $I$, it follows that

$$
\begin{aligned}
|x| \leq \varphi\left(\left|x_{0}\right|, 1\right) & =\left|x_{0}\right|^{1 / 2} \ln ^{1 / 2}\left(1+\left|x_{0}\right|\right) \\
& \leq\left|x_{0}\right|^{1 / 2} \ln ^{1 / 2}\left(1+\left|x_{0}\right| u^{2}\right)=\varphi\left(\left|x_{0}\right| u, \frac{1}{u}\right),
\end{aligned}
$$

and so $x \in \varphi\left(L_{1 / u}^{1}, L_{u}^{\infty}\right)$ with norm $\leq 1$. Thus we have a continuous imbedding

$$
\varphi\left(L^{1}, L^{\infty}\right) \stackrel{1}{\hookrightarrow} \varphi\left(L_{1 / u}^{1}, L_{u}^{\infty}\right)
$$

As concrete weight $\mathrm{u}$ on $I=(0,1)$ for which $\int_{0}^{1} u(t) d t \leq 2$ and $u(t) \geq 1$ for all $t \in(0,1)$ we can take $u(t)=t^{-1 / 2}$ on $(0,1)$. 


\section{Factorization of Positive Sublinear Operators in $X^{(p)}$ Spaces}

Let $X$ be either $L^{0}(\mu)$ or a Banach ideal space on $(\Omega, \mu)$. An operator $T: X \rightarrow L^{0}(\nu)$ is called positive if $0 \leq x \in X$ implies that $0 \leq T x \in L^{0}(\nu)$; $T$ is called sublinear if, for all $x, y \in X$ and any $a \in \mathbf{R}$,

$$
|T(x+y)(t)| \leq|T x(t)|+|T y(t)| \text { and }|T(a x)(t)|=|a||T x(t)| \quad \nu \text {-a.e. }
$$

Classical examples of positive linear operators are integral operators $K x(t)=$ $\int_{\Omega} k(s, t) x(s) d s$ with positive measurable kernels $k(s, t) \geq 0$ and as positive sublinear operators we can take maximal operators and $L x(t)=\int_{\Omega}|l(s, t)| x(s) d s$ with measurable kernel $l(s, t)$.

If $1 \leq p<\infty$ and $1 / p+1 / p^{\prime}=1$, then for any positive sublinear operator $T$ the pointwise Hölder-Rogers ${ }^{1}$ inequality is true:

$$
T\left(|x|^{1 / p}|y|^{1-1 / p}\right)(t) \leq[T(|x|)(t)]^{1 / p}[T(|y|)(t)]^{1-1 / p} \quad \nu \text {-a.e. }
$$

which can be rewritten as

$$
T(|x||y|)(t) \leq\left[T\left(|x|^{p}\right)(t)\right]^{1 / p}\left[T\left(|y|^{p^{\prime}}\right)(t)\right]^{1 / p^{\prime}} \quad \nu \text {-a.e. }
$$

for any $x, y \in X$. This estimate follows directly from the equality

$$
a^{1 / p} b^{1-1 / p}=\inf _{\varepsilon>0}\left[\frac{1}{p} \varepsilon^{\frac{1}{p}-1} a+\left(1-\frac{1}{p}\right) \varepsilon^{\frac{1}{p}} b\right],
$$

which is true for any real positive numbers $a, b$. Note that more general pointwise estimates for positive sublinear operators can be proved. In fact, this was used (but not explicitly written) for positive linear operators in the proof of the fact that the Calderón-Lozanovskil spaces are exact interpolation spaces for positive linear operators (see [3, 32, 50]; see also [35, Theorem 15.13]). It was also noted in [37] that the same estimate is true for positive sublinear operators. We include the proof here.

Lemma 4 Let $X$ be either $L^{0}(\mu)$ or a Banach ideal space on $(\Omega, \mu)$ and let $T: X \rightarrow$ $L^{0}(\nu)$ be a positive sublinear operator. If $\varphi \in \mathcal{U}$, then for any $x, y \in X$

$$
T(\varphi(|x|,|y|))(t) \leq \varphi(T(|x|)(t), T(|y|)(t)) \quad \nu \text {-a.e. }
$$

Proof Since for arbitrary $a>0, b>0$,

$$
\varphi(|x|,|y|) \leq \frac{a|x|+b|y|}{\hat{\varphi}(a, b)}
$$

it follows that

$$
T(\varphi(|x|,|y|)) \leq \frac{a T(|x|)+b T(|y|)}{\hat{\varphi}(a, b)}
$$

\footnotetext{
${ }^{1}$ The classical Hölder inequality should historically correctly be called the Hölder-Rogers inequality (cf. [36]).
} 
for arbitrary $a, b>0$, and so

$$
T(\varphi(|x|,|y|)) \leq \hat{\hat{\varphi}}(T(|x|), T(|y|))=\varphi(T(|x|), T(|y|))
$$

Lemma 5 Let $X$ be either $L^{0}(\mu)$ or a Banach ideal space on $(\Omega, \mu)$. Assume that $T: X \rightarrow L^{0}(\nu)$ is a positive sublinear operator. Then, for any weights $w_{0}, w_{1}$ on $\Omega$ and $1 \leq p<\infty$, the operator defined by

$$
T_{p} x(t)=\left[w_{0}(t) T\left(|x|^{p} w_{1}\right)(t)\right]^{1 / p}
$$

is positive and sublinear.

Proof We have, by using the Hölder-Rogers inequality (8) similarly as in the proof of the Minkowski inequality,

$$
\begin{aligned}
{\left[T_{p}(x+y)(t)\right]^{p} \leq } & w_{0}(t) T\left(|x+y|^{p} w_{1}\right)(t) \\
\leq & w_{0}(t) T\left(|x||x+y|^{p-1} w_{1}+|y||x+y|^{p-1} w_{1}\right)(t) \\
\leq & w_{0}(t) T\left(|x||x+y|^{p-1} w_{1}\right)+T\left(|y||x+y|^{p-1} w_{1}\right)(t) \\
\leq & w_{0}(t) T\left(|x|^{p} w_{1}\right)^{1 / p} T\left(|x+y|^{(p-1) p^{\prime}} w_{1}\right)^{1 / p^{\prime}} \\
& \quad+w_{0}(t) T\left(|y|^{p} w_{1}\right)^{1 / p} T\left(|x+y|^{(p-1) p^{\prime}} w_{1}\right)^{1 / p^{\prime}} \\
= & w_{0}(t) T\left(|x|^{p} w_{1}\right)^{1 / p} T\left(|x+y|^{p} w_{1}\right)^{1 / p^{\prime}} \\
& \quad+w_{0}(t) T\left(|y|^{p} w_{1}\right)^{1 / p} T\left(|x+y|^{p} w_{1}\right)^{1 / p^{\prime}} \\
= & {\left.\left[T_{p}(x)(t)+T_{p}(y)(t)\right] T_{p}(x+y)(t)\right]^{p / p^{\prime}} }
\end{aligned}
$$

which gives the subadditivity of $T_{p}$. Moreover, $T_{p}(a x)(t)=|a| T_{p}(x)(t)$ and the proof is complete.

Let us start with a result to which the idea in the $L^{p}$-spaces was given by Gagliardo [15, Lemma 3.I] and by Rubio de Francia [48].

Lemma 6 Let $X$ be a Banach ideal space on $(\Omega, \mu)$ and let $T: X \rightarrow X$ be a bounded positive sublinear operator. Then there exists $u \in X, u(t)>0 \mu$-a.e. on $\Omega$ such that $T u(t) \leq C u(t)$ for $t \in \Omega \mu$-a.e., where $C=(1+\varepsilon)\|T\|_{X \rightarrow X}$ with any $\varepsilon>0 .{ }^{2}$

Proof Take $x_{0} \in X$ with $x_{0}(t)>0$ for $t \in \Omega \mu$-a.e. and put

$$
u(t)=\sum_{k=0}^{\infty} C^{-k} T^{k} x_{0}(t), \quad \text { where } T^{0}=\mathrm{Id} .
$$

\footnotetext{
${ }^{2}$ As usual, for the norm $\|T\|_{X \rightarrow X}$ of a sublinear operator $T$ we mean $\sup _{\|x\|_{X} \leq 1}\|T x\|_{X}$.
} 
Since

$$
\sum_{k=0}^{\infty} C^{-k}\left\|T^{k} x_{0}\right\|_{X} \leq \sum_{k=0}^{\infty} C^{-k}\|T\|_{X \rightarrow X}^{k}\left\|x_{0}\right\|_{X}=\sum_{k=0}^{\infty}(1+\varepsilon)^{-k}\left\|x_{0}\right\|_{X}=\left(1+\frac{1}{\varepsilon}\right)\left\|x_{0}\right\|_{X}
$$

it follows that $u \in X$ and $\|u\|_{X} \leq\left(1+\frac{1}{\varepsilon}\right)\left\|x_{0}\right\|_{X}$. Moreover, by the positivity of the operator $T$, we have

$$
0<x_{0}(t) \leq x_{0}(t)+T x_{0}(t) / C+T^{2} x_{0}(t) / C^{2}+\cdots=u(t) \quad \text { for } t \in \Omega \mu \text {-a.e. }
$$

and

$$
\begin{aligned}
T u(t) & \leq \sum_{k=0}^{\infty} C^{-k} T^{k+1} x_{0}(t)=C \sum_{k=1}^{\infty} C^{-k} T^{k} x_{0}(t) \\
& \leq C\left[x_{0}(t)+\sum_{k=1}^{\infty} C^{-k} T^{k} x_{0}(t)\right]=C u(t) .
\end{aligned}
$$

Now we are ready to state and prove the fundamental factorization theorem in weighted Banach ideal $X^{(p)}$ spaces.

Theorem 5 For some weight functions $v_{0}, v_{1}, w_{0}, w_{1}$ on $\Omega$ and some $p_{0}, p_{1}, q_{0}, q_{1} \in$ $[1, \infty)$, let the operators $T_{0}:\left(X_{v_{0}}\right)^{\left(p_{0}\right)} \rightarrow\left(X_{v_{1}}\right)^{\left(p_{1}\right)}$ and $T_{1}:\left(X_{w_{0}}\right)^{\left(q_{0}\right)} \rightarrow\left(X_{w_{1}}\right)^{\left(q_{1}\right)}$ be positive sublinear and bounded with the corresponding norms $C_{0}$ and $C_{1}$. Assume that we have continuous imbeddings $X^{\left(p_{1}\right)} \subset X^{\left(p_{0}\right)}$ and $X^{\left(q_{1}\right)} \subset X^{\left(q_{0}\right)}$ with the norms not exceeding $C_{2}$ and $C_{3}$, respectively. Then:

(i) There exists a positive weight $u \in X^{\left(p_{0} q_{0}\right)}$ such that

$$
v_{1} T_{0}\left(u^{q_{0}} v_{0}^{-1}\right) \leq C^{q_{0}} u^{q_{0}} \text { and } w_{1} T_{1}\left(u^{p_{0}} w_{0}^{-1}\right) \leq C^{p_{0}} u^{p_{0}}
$$

with $C=2\left(C_{0}^{1 / q_{0}} C_{2}^{1 / q_{0}}+C_{1}^{1 / p_{0}} C_{3}^{1 / p_{0}}\right)$ or, equivalently, we have that

$$
T_{0}: L_{v_{0} u^{-q_{0}}}^{\infty} \rightarrow L_{v_{1} u^{-q_{0}}}^{\infty} \text { and } T_{1}: L_{w_{0} u^{-p_{0}}}^{\infty} \rightarrow L_{w_{1} u^{-p_{0}}}^{\infty}
$$

are bounded with norms not exceeding $C^{q_{0}}$ and $C^{p_{0}}$, respectively.

(ii) There exists a positive weight $u \in X^{\left(p_{1} q_{1}\right)}$ such that

$$
v_{1} T_{0}\left(u^{q_{1}} v_{0}^{-1}\right) \leq D^{q_{1}} u^{q_{1}} \text { and } w_{1} T_{1}\left(u^{p_{1}} w_{0}^{-1}\right) \leq D^{p_{1}} u^{p_{1}}
$$

with $D=2\left(C_{0}^{1 / q_{1}} C_{2}^{1 / q_{1}}+C_{1}^{1 / p_{1}} C_{3}^{1 / p_{1}}\right)$ or, equivalently, we have that

$$
T_{0}: L_{v_{0} u^{-q_{1}}}^{\infty} \rightarrow L_{v_{1} u^{-q_{1}}}^{\infty} \text { and } T_{1}: L_{w_{0} u^{-p_{1}}}^{\infty} \rightarrow L_{w_{1} u^{-p_{1}}}^{\infty}
$$

are bounded with norms not exceeding $D^{q_{1}}$ and $D^{p_{1}}$, respectively. 
Proof (i) Using the given operator $T_{0}$ we can construct a new positive sublinear operator $S_{0}$ by

$$
S_{0} x=\left[v_{1} T_{0}\left(|x|^{q_{0}} v_{0}^{-1}\right)\right]^{1 / q_{0}} .
$$

Of course, $S_{0}$ is positive, and by Lemma 5 it is sublinear. The operator $S_{0}$ is also bounded from $X^{\left(p_{0} q_{0}\right)}$ into $X^{\left(p_{1} q_{0}\right)}$ with the norm $\leq C_{0}^{1 / q_{0}}$. Indeed,

$$
\begin{aligned}
\left\|S_{0} x\right\|_{X^{\left(p_{1} q_{0}\right)}} & =\left\|\left[v_{1} T_{0}\left(|x|^{q_{0}} v_{0}^{-1}\right)\right]^{p_{1}}\right\|_{X}^{\frac{1}{p_{1} q_{0}}} \\
& \leq C_{0}^{1 / q_{0}}\left\|\left[v_{0}|x|^{q_{0}} v_{0}^{-1}\right]^{p_{0}}\right\|_{X}^{\frac{1}{p_{0} q_{0}}} \\
& =C_{0}^{1 / q_{0}}\left\||x|^{p_{0} q_{0}}\right\|_{X}^{\frac{1}{p_{0} q_{0}}}=C_{0}^{1 / q_{0}}\|x\|_{X^{\left(p_{0} q_{0}\right)}} .
\end{aligned}
$$

Similarly, the operator $S_{1}$ given by

$$
S_{1} x=\left[w_{1} T_{1}\left(|x|^{p_{0}} w_{0}^{-1}\right)\right]^{1 / p_{0}}
$$

is positive, sublinear and bounded from $X^{\left(p_{0} q_{0}\right)}$ into $X^{\left(p_{0} q_{1}\right)}$ with norm $\leq C_{1}^{1 / p_{0}}$.

Since we have imbeddings $X^{\left(p_{1} q_{0}\right)} \subset X^{\left(p_{0} q_{0}\right)}$ and $X^{\left(p_{0} q_{1}\right)} \subset X^{\left(p_{0} q_{0}\right)}$, it follows that the operator $S=S_{0}+S_{1}$ is bounded from $X^{\left(p_{0} q_{0}\right)}$ into $X^{\left(p_{0} q_{0}\right)}$ with norm $\leq C$, and applying Lemma 6 to $S$ we obtain the required estimates.

(ii) The proof here is similar. We should only consider the operators

$$
L_{0} x=\left[v_{1} T_{0}\left(|x|^{q_{1}} v_{0}^{-1}\right)\right]^{1 / q_{1}} \text { and } L_{1} x=\left[w_{1} T_{1}\left(|x|^{p_{1}} w_{0}^{-1}\right)\right]^{1 / p_{1}},
$$

use the embeddings $X^{\left(p_{1} q_{1}\right)} \subset X^{\left(p_{0} q_{1}\right)}, X^{\left(p_{1} q_{1}\right)} \subset X^{\left(p_{1} q_{0}\right)}$ and apply Lemma 6 to the operator $L=L_{0}+L_{1}$ which is bounded from $X^{\left(p_{1} q_{1}\right)}$ into itself. The proof is complete.

In some cases we do not need the above imbeddings. We can then formulate a generalization to $X^{(p)}$ spaces of the result of Rubio de Francia type. This result gives the factorization theorem through weighted $L^{\infty}$ spaces.

Corollary 5 Assume that for some weight functions $v, w$ on $\Omega$ and some $p_{0}, p_{1} \in$ $[1, \infty)$ the operators

$$
T_{0}:\left(X_{v}\right)^{\left(p_{0}\right)} \rightarrow\left(X_{v}\right)^{\left(p_{0}\right)} \text { and } T_{1}:\left(X_{w}\right)^{\left(p_{1}\right)} \rightarrow\left(X_{w}\right)^{\left(p_{1}\right)}
$$

are positive, sublinear and bounded with the corresponding norms $C_{0}$ and $C_{1}$. Then there exists a positive weight $u \in X^{\left(p_{0} p_{1}\right)}$ such that

$$
v T_{0}\left(u^{p_{1}} v^{-1}\right) \leq C^{p_{1}} u^{p_{1}} \text { and } w T_{1}\left(u^{p_{0}} w^{-1}\right) \leq C^{p_{0}} u^{p_{0}},
$$

with $C=2\left(C_{0}^{1 / p_{1}}+C_{1}^{1 / p_{0}}\right)$. The last estimates mean that the operators

$$
T_{0}: L_{v u^{-p_{1}}}^{\infty} \rightarrow L_{v u^{-p_{1}}}^{\infty} \text { and } T_{1}: L_{w u^{-p_{0}}}^{\infty} \rightarrow L_{w u^{-p_{0}}}^{\infty}
$$

are bounded with norms not exceeding $C^{p_{1}}$ and $C^{p_{0}}$, respectively. 
As special cases of Theorem 5 and Corollary 5 we obtain the factorization results of Hernández (see [17, Theorem 2.1] and [18, Theorem 1]).

Corollary 6 If $T_{0}: L^{p_{0}} \rightarrow L^{p_{0}}$ and $T_{1}: L^{p_{1}} \rightarrow L^{p_{1}}$ are bounded, positive sublinear operators, then there exists a positive weight $u \in L^{p_{0} p_{1}}$ such that $T_{0} u^{p_{1}} \leq C u^{p_{1}}$ and $T_{1} u^{p_{0}} \leq C u^{p_{0}}$ or, equivalently, we have that the operators $T_{0}: L_{u^{-p_{1}}}^{\infty} \rightarrow L_{u^{-p_{1}}}^{\infty}$ and $T_{1}: L_{u^{-p_{0}}}^{\infty} \rightarrow L_{u^{-p_{0}}}^{\infty}$ are bounded.

In Theorem 5 and Corollaries 5 and 6 there are two operators $T_{0}$ and $T_{1}$ but in applications sometimes as an operator $T_{1}$ is taken the associated operator $T_{0}^{\prime}$ (sometimes also called the dual operator in the sense of Köthe) to $T_{0}$. If $T_{0} \in \mathcal{K}$, then the associated operator does not always exist. Here by $\mathcal{K}$ we denote the class of positive sublinear operators $T$ defined on $L^{0}(\mu)$ with values in $L^{0}(\mu)$ and for $T \in \mathcal{K}$ we consider the notion of the associated operator $T^{\prime} \in \mathcal{K}$.

For $T \in \mathcal{K}$, an operator $T^{\prime} \in \mathcal{K}$ is called associated to $T$ (in the scale of $L^{p}$-spaces) if, for all $1 \leq p \leq \infty$ and all weights $u$ we have that $T: L_{u}^{p} \rightarrow L_{u}^{p}$ is bounded if and only if $T^{\prime}: L_{1 / u}^{p^{\prime}} \rightarrow L_{1 / u}^{p^{\prime}}$ is bounded, and the estimates

$$
\frac{1}{C}\|T\|_{L_{u}^{p} \rightarrow L_{u}^{p}} \leq\left\|T^{\prime}\right\|_{L_{1 / u}^{p^{\prime} \rightarrow L_{1 / u}^{p^{\prime}}}} \leq C\|T\|_{L_{u}^{p} \rightarrow L_{u}^{p}}
$$

hold with a constant $C>0$ independent of $p$ and $u$.

Note that $T^{\prime}$ is not necessary unique. If $T$ is a linear operator, then as $T^{\prime}$ we can take the conjugate operator $T^{*}$. Also for a linear operator $T$ the operator $x \mapsto|T x|$ is sublinear and there is no notion of conjugate operator to it but we can instead take $T^{\prime} x=\left|T^{*} x\right|$.

We are now ready to formulate the factorization theorem in $L^{p}$-spaces with the factorization through the weighted $L^{1}$ and $L^{\infty}$ spaces for operators $T \in \mathcal{K}$ for which an associated operator $T^{\prime} \in \mathcal{K}$ exists.

Corollary 7 Let $1<p<\infty$. Assume that $T \in \mathcal{K}$ and for $T$ there exists $T^{\prime} \in \mathcal{K}$. Then $T: L^{p} \rightarrow L^{p}$ is bounded if and only if there exists a weight $u \in L^{p}$ on $\Omega$ such that

$$
T: L_{u^{p-1}}^{1} \rightarrow L_{u^{p-1}}^{1} \text { and } T: L_{1 / u}^{\infty} \rightarrow L_{1 / u}^{\infty}
$$

is bounded.

Proof If $T: L^{p} \rightarrow L^{p}$ and $T^{\prime}: L^{p^{\prime}} \rightarrow L^{p^{\prime}}$ are bounded then, by Corollary 6, there exists $w \in L^{p p^{\prime}}$ such that

$$
T w^{p^{\prime}} \leq C w^{p^{\prime}} \text { and } T^{\prime} w^{p} \leq C w^{p}
$$

Taking $u=w^{p^{\prime}}$ we have $u \in L^{p}$ and

$$
T u \leq C u \text { and } T^{\prime} u^{p-1} \leq C u^{p-1}
$$

or

$$
T: L_{1 / u}^{\infty} \rightarrow L_{1 / u}^{\infty} \text { and } T: L_{u^{p-1}}^{1} \rightarrow T: L_{u^{p-1}}^{1}
$$


is bounded.

Conversely, if $T$ is bounded in $L_{u^{p-1}}^{1}$ and in $L_{1 / u^{\prime}}^{\infty}$, then $T$ is also bounded in the Calderón spaces $\left(L_{u^{p-1}}^{1}\right)^{1 / p}\left(L_{1 / u}^{\infty}\right)^{1-1 / p}=L^{p}$.

We will show a little later that if for an operator $T \in \mathcal{K}$ we do not put additional restrictions, (for example, the existence of an associated operator) then the factorization theorem through weighted $L^{1}$ and $L^{\infty}$ spaces cannot be true.

Before giving this counter-example we would like to show that for some class of operators we can prove a factorization theorem where the extreme spaces are Lorentz and Marcinkiewicz spaces determined by weight instead of weighted Lebesgue spaces $L^{1}$ and $L^{\infty}$. Lorentz and Marcinkiewicz spaces are natural extreme spaces in the class of symmetric spaces, $c f$. [25]. All our spaces here are on $(0, \infty)$.

We consider a subclass $\mathcal{K}_{*}$ of operators $T \in \mathcal{K}$ for which there exists a constant $C>0$ such that

$$
\int_{0}^{t}(T x)^{*}(s) d s \leq C \int_{0}^{t} T x^{*}(s) d s
$$

for all $t>0$ and $x \in L^{0}(0, \infty)$.

As an example of $T \in \mathcal{K}_{*}$ we can take the Hardy operator $H x(t)=\frac{1}{t} \int_{0}^{t} x(s) d s$, Hardy sublinear operator $H^{*} x(t)=\frac{1}{t} \int_{0}^{t} x^{*}(s) d s$, maximal operator $M$, and integral operator $T x(t)=\int_{0}^{\infty} k(t, s) x(s) d s$ with a positive kernel $k(t, s) \geq 0$ which is decreasing in each variable separately.

We recall the definition of Lorentz $\Lambda_{u^{*}}$ spaces and Marcinkiewicz $M_{u^{*}}$ spaces. For the weight function $u$ on $(0, \infty)$, the Lorentz space $\Lambda_{u^{*}}$ is the space generated by the norm

$$
\|x\|_{\Lambda_{u^{*}}}=\int_{0}^{\infty} x^{*}(t) u^{*}(t) d t
$$

and the Marcinkiewicz space $M_{u^{*}}$ that by the norm

$$
\|x\|_{M_{u^{*}}}=\sup _{t>0} \frac{1}{\int_{0}^{t} u^{*}(s) d s} \int_{0}^{t} x^{*}(s) d s .
$$

Theorem 6 Let $1<p<\infty$. Assume that $T \in \mathcal{K}_{*}$ and for $T$ there exists an associated operator $T^{\prime} \in \mathcal{K}$. If $T: L^{p} \rightarrow L^{p}$ is bounded, then there exists a positive weight $u \in L^{p}$ such that

(i) The estimates

$$
\frac{1}{t} \int_{0}^{t} u^{*}(s) d s \leq C_{1} u(t), \frac{1}{t} \int_{0}^{t} u^{*}(s)^{p-1} d s \leq C_{2} u(t)^{p-1}
$$

and

$$
\int_{t}^{\infty} \frac{u^{*}(s)}{s} d s \leq C_{3} u(t), \int_{t}^{\infty} \frac{u^{*}(s)^{p-1}}{s} d s \leq C_{4} u(t)^{p-1}
$$

hold for all $t>0$.

(ii) The operators $T: \Lambda_{u^{* p-1}} \rightarrow \Lambda_{u^{* p-1}}$ and $T: M_{u^{*}} \rightarrow M_{u^{*}}$ are bounded.

Conversely, if conditions (i) and (ii) are satisfied then $T: L^{p} \rightarrow L^{p}$ is bounded. 
Proof Assume that $T: L^{p} \rightarrow L^{p}$ is bounded. Consider two operators

$$
S_{0}=T+H^{*}+H_{*}: L^{p} \rightarrow L^{p} \text { and } S_{1}=T^{\prime}+H^{*}+H_{*}: L^{p^{\prime}} \rightarrow L^{p^{\prime}}
$$

where $H^{*} x(t)=x^{* *}(t)=\frac{1}{t} \int_{0}^{t} x^{*}(s) d s$ and $H_{*} x(t)=\int_{t}^{\infty} \frac{x^{*}(s)}{s} d s$.

Note that $H^{*}$ is bounded in $L^{p}$ spaces for all $1<p \leq \infty$ and $H_{*}$ is bounded in $L^{p}$ spaces for all $1 \leq p<\infty$. Then $S_{0}, S_{1} \in \mathcal{K}$ and by Corollary 6 we can find a weight $w \in L^{p p^{\prime}}$ such that the operators

$$
S_{0}: L_{w^{-p^{\prime}}}^{\infty} \rightarrow L_{w^{-p^{\prime}}}^{\infty} \text { and } S_{1}: L_{w^{-p}}^{\infty} \rightarrow L_{w^{-p}}^{\infty}
$$

are bounded, which can be rewritten by taking $u=w^{p^{\prime}}$ such that $u \in L^{p}$ and

$$
S_{0}: L_{1 / u}^{\infty} \rightarrow L_{1 / u}^{\infty} \text { and } S_{1}: L_{u^{1-p}}^{\infty} \rightarrow L_{u^{1-p}}^{\infty}
$$

are bounded. Since $H^{*}: L_{1 / u}^{\infty} \rightarrow L_{1 / u}^{\infty}$ is bounded with norm $\leq A$, it follows that

$$
H^{*} u(t)=u^{* *}(t) \leq A u(t)
$$

for all $t>0$, and the first estimate in (i) is proved.

The operator $T: L_{1 / u}^{\infty} \rightarrow L_{1 / u}^{\infty}$ is also bounded with norm $\leq B$. Therefore $|T u(t)| \leq B u(t)$ for all $t>0$, and so

$$
(T u)^{*}(t) \leq B u^{*}(t) \quad \text { for all } t>0
$$

If we assume that $x^{* *}(t) \leq u^{* *}(t)$ for all $t>0$, then by the assumption $T \in \mathcal{K}_{*}$ we obtain

$$
\begin{aligned}
(T x)^{* *}(t) & =\frac{1}{t} \int_{0}^{t}(T x)^{*}(s) d s \leq \frac{C}{t} \int_{0}^{t} T x^{*}(s) d s \\
& \leq \frac{C}{t} \int_{0}^{t} T u^{* *}(s) d s \leq \frac{A C}{t} \int_{0}^{t} T u(s) d s \leq \frac{A C}{t} \int_{0}^{t}(T u)^{*}(s) d s \\
& \leq \frac{A B C}{t} \int_{0}^{t} u^{*}(s) d s=A B C u^{* *}(t),
\end{aligned}
$$

and so $T: M_{u^{*}} \rightarrow M_{u^{*}}$ is bounded with the norm $\leq A B C$.

We also have that $H^{*}: L_{u^{1-p}}^{\infty} \rightarrow L_{u^{1-p}}^{\infty}$ is bounded with the norm $\leq D$ which gives the second estimate in (i)

$$
\frac{1}{t} \int_{0}^{t} u^{*}(s)^{p-1} d s \leq D u(t)^{p-1} \quad \text { for all } t>0
$$

The operator $T \in \mathcal{K}_{*}$ satisfies the estimate $\int_{0}^{t}(T x)^{*}(s) d s \leq C \int_{0}^{t} T x^{*}(s) d s$ for all $t>0$. Therefore, by the well-known property of rearrangement (see [25], property 
$18^{0}$ ) and the boundedness of $T$ in $L_{u^{p-1}}^{1}$ with the norm $\leq E$, we obtain

$$
\begin{aligned}
\|T x\|_{\Lambda_{u^{*} p-1}} & =\int_{0}^{\infty} u^{*}(t)^{p-1}(T x)^{*}(t) d t \\
& \leq C \int_{0}^{\infty} u^{*}(t)^{p-1} T x^{*}(t) d t \leq C D \int_{0}^{\infty} u(t)^{p-1} T x^{*}(t) d t \\
& \leq C D E \int_{0}^{\infty} u(t)^{p-1} x^{*}(t) d t \leq C D E \int_{0}^{\infty} u^{*}(t)^{p-1} x^{*}(t) d t \\
& =C D E\|x\|_{\Lambda_{u^{*}-1}},
\end{aligned}
$$

and $T: \Lambda_{u^{* p-1}} \rightarrow \Lambda_{u^{* p-1}}$ is bounded with the norm $\leq C D E$.

The boundedness of $H_{*}: L_{1 / u}^{\infty} \rightarrow L_{1 / u}^{\infty}$ and $H_{*}: L_{u^{1-p}}^{\infty} \rightarrow L_{u^{1-p}}^{\infty}$ gives the third and the fourth estimate in (i).

Assume that conditions (i) and (ii) are satisfied. Then it is enough to show that any $L^{p}$ space can be described from $\Lambda_{u^{*} p-1}$ and $M_{u^{*}}$ by the real method of interpolation (the $K$-method of interpolation). We have

$$
M_{u^{*}}=\left(L^{1}, L^{\infty}\right)_{\Phi_{1} ; K} \text { with } \Phi_{1}=L_{\frac{1}{v}}^{\infty}, v(t)=\int_{0}^{t} u^{*}(s) d s
$$

and the first with the third estimate in (i) ensures that $\Phi_{1}$ is a quasi-power parameter, that is, the Calderón operator

$$
S f(t)=\int_{0}^{\infty} \min \left(1, \frac{t}{s}\right)|f(s)| \frac{d s}{s}
$$

is bounded in $L_{1 / v}^{\infty}$ (see [10, p. 387] for the definition and examples). In fact,

$$
\begin{aligned}
S f(t) & =\int_{0}^{t}|f(s)| \frac{d s}{s}+t \int_{t}^{\infty}|f(s)| \frac{d s}{s^{2}} \\
& \leq\|f\|_{L_{\frac{1}{v}}^{\infty}}\left(\int_{0}^{t} v(s) \frac{d s}{s}+t \int_{t}^{\infty} v(s) \frac{d s}{s^{2}}\right) \\
& =\|f\|_{L_{\frac{1}{v}}^{\infty}}\left(\int_{0}^{t} u^{* *}(s) d s+t \int_{t}^{\infty} u^{* *}(s) \frac{d s}{s}\right) \\
& \leq C_{1}\|f\|_{L_{\frac{1}{v}}^{\infty}}\left(\int_{0}^{t} u^{*}(s) d s+t \int_{t}^{\infty} \frac{u^{*}(s)}{s} d s\right) \\
& \leq C_{1}\|f\|_{L_{\frac{1}{v}}^{\infty}}\left(v(t)+C_{3} t u^{*}(t)\right) \leq C_{1}\left(1+C_{3}\right)\|f\|_{L_{\frac{1}{v}}^{\infty}} v(t) .
\end{aligned}
$$

The second and the fourth estimate in (i) ensure that $\Phi_{0}=L_{\frac{u^{*}(t)^{p-1}}{t}}^{1}$ is a quasi-power 
parameter. In fact,

$$
\begin{aligned}
\int_{0}^{\infty} \frac{u^{*}(t)^{p-1}}{t} S f(t) d t & =\int_{0}^{\infty} \frac{u^{*}(t)^{p-1}}{t}\left(\int_{0}^{t}|f(s)| \frac{d s}{s}+t \int_{t}^{\infty}|f(s)| \frac{d s}{s^{2}}\right) d t \\
= & \int_{0}^{\infty}\left(\int_{s}^{\infty} \frac{u^{*}(t)^{p-1}}{t} d t\right)|f(s)| \frac{d s}{s} \\
& \quad+\int_{0}^{\infty}\left(\int_{0}^{s} u^{*}(t)^{p-1} d t\right)|f(s)| \frac{d s}{s^{2}} \\
\leq & C_{4} \int_{0}^{\infty} u^{*}(s)^{p-1}|f(s)| \frac{d s}{s}+C_{2} \int_{0}^{\infty} u^{*}(s)^{p-1}|f(s)| \frac{d s}{s} \\
& =\left(C_{2}+C_{4}\right)\|f\|_{L^{1}}
\end{aligned}
$$

We also have

$$
\Lambda_{u^{* p-1}}=\left(L^{1}, L^{\infty}\right)_{\Phi_{0} ; K}
$$

The last identification of the spaces follows from the estimates

$$
\begin{aligned}
\|x\|_{\Lambda_{u^{*} p-1}} & =\int_{0}^{\infty} x^{*}(t) u^{*}(t)^{p-1} d t \\
& \leq \int_{0}^{\infty}\left(\int_{0}^{t} x^{*}(s) d s\right) u^{*}(t)^{p-1} \frac{d t}{t} \\
& =\|x\|_{\Phi_{0} ; K}=\int_{0}^{\infty}\left(\int_{s}^{\infty} \frac{u^{*}(t)^{p-1}}{t} d t\right) x^{*}(s) d s \\
& \leq C_{4} \int_{0}^{\infty} u^{*}(s)^{p-1} x^{*}(s) d s=C_{4}\|x\|_{\Lambda_{u^{*} p-1}}
\end{aligned}
$$

Using now a generalization of the Holmstedt formula with quasi-power parameters $\Phi_{0}, \Phi_{1}$, proved by Dmitriev-Ovchinnikov (1979) and Brudnyı̆-Krugljak (1981) (see $[10$, Corollary 7.1.1, p. 466] and [41, p. 30] in the discrete case),

$$
K\left(t, a ;\left(A_{0}, A_{1}\right)_{\Phi_{0} ; K},\left(A_{0}, A_{1}\right)_{\Phi_{1} ; K}\right) \approx K\left(t, K\left(\cdot, a ; A_{0}, A_{1}\right) ; \Phi_{0}, \Phi_{1}\right)
$$

we obtain

$$
\begin{aligned}
K\left(t, x ; \Lambda_{u^{* p-1}}, M_{u^{*}}\right) & \approx K\left(t, x:\left(L^{1}, L^{\infty}\right)_{\Phi_{0} ; K},\left(L^{1}, L^{\infty}\right)_{\Phi_{1} ; K}\right) \\
& \approx K\left(t, K\left(s, x ; L^{1}, L^{\infty}\right) ; L_{\frac{u^{*}(s) p-1}{s}}^{1}, L_{\frac{1}{s u^{*}(s)}}^{\infty}\right) \\
& \approx K\left(t, \int_{0}^{s} x^{*}(u) d u ; L_{\frac{u^{*}(s) p-1}{s}}^{1}, L_{\frac{1}{s u^{*}(s)}}^{\infty}\right) \\
& =K\left(t, x^{* *}(s) ; L_{u^{* p-1}}^{1}, L_{\frac{1}{u^{*}}}^{\infty}\right)
\end{aligned}
$$


Thus, for $\theta=1-\frac{1}{p}$,

$$
\begin{aligned}
\|x\|_{\left(\Lambda_{u^{*} p-1}, M_{u^{*}}\right)_{\theta, p}} & \approx\left\|x^{* *}\right\|_{\left(L_{u^{* p-1}}^{1}, L_{\frac{1}{u^{*}}}^{\infty}\right)_{\theta, p}} \\
& \approx\left\|x^{* *}\right\|_{L_{w}^{p}}=\left\|x^{* *}\right\|_{L^{p}} \approx\left\|x^{*}\right\|_{L^{p}}=\|x\|_{L^{p}},
\end{aligned}
$$

where the first equality comes from the fact that

$$
w(t)=\left(u^{*}(t)^{p-1}\right)^{1-\theta}\left(\frac{1}{u^{*}(t)}\right)^{\theta}=u^{*}(t)^{1-1 / p}\left(\frac{1}{u^{*}(t)}\right)^{1-1 / p}=1 .
$$

Thus $\left(\Lambda_{u^{* p-1}}, M_{u^{*}}\right)_{\theta, p}=L^{p}$ with equivalent norms and the proof is complete.

Corollary 8 If $1<p<\infty$ and estimates in (i) are satisfied, then $\left(\Lambda_{u^{* p-1}}, M_{u^{*}}\right)_{\theta, p}=$ $L^{p}$ and so $L^{p}$ is an interpolation space between $\Lambda_{u^{* p-1}}$ and $M_{u^{*}}$. In particular, since $u(t)=t^{-1 / p}$ with $1<p<\infty$ satisfies estimates in (i) we obtain $\left(L^{p, 1}, L^{p, \infty}\right)_{1-1 / p, p}=$ $L^{p}$ and so $L^{p}$ is an interpolation space between Lorentz space $L^{p, 1}$ and the Marcinkiewicz weak $L^{p}$-space $L^{p, \infty}$.

Let us consider the factorization theorem of Schur type, that is, a factorization through weighted $L^{1}$ and $L^{\infty}$ spaces. We will show the failure of a Schur type factorization theorem even for powers in symmetric spaces for the positive sublinear Hardy operator.

A symmetric space $X$ on $(0, \infty)$ is a Banach ideal space on $(0, \infty)$ with the additional property that $x^{*}(t) \leq y^{*}(t)$ for every $t>0$ and $y \in X$ imply $x \in X$ and $\|x\|_{X} \leq\|y\|_{X}$, where $x^{*}$ denotes the decreasing rearrangement of $|x|$ (see [25] for definition and properties). The fundamental function $\varphi_{X}(t)$ of $X$ is defined by $\varphi_{X}(t)=\left\|\chi_{(0, t)}\right\|_{X}, t>0$.

Given $\lambda>0$, the dilation operator $\sigma_{\lambda}$ given by $\sigma_{\lambda} x(t)=x(t / \lambda), t>0$, is well defined in every symmetric space $X$ and $\left\|\sigma_{\lambda}\right\|_{X \rightarrow X} \leq \max (1, \lambda)$. The classical Boyd indices of $X$ are defined by $(c f .[2,25,27])$

$$
\alpha_{X}=\lim _{\lambda \rightarrow 0} \frac{\ln \left\|\sigma_{\lambda}\right\|_{X \rightarrow X}}{\ln \lambda}, \beta_{X}=\lim _{\lambda \rightarrow \infty} \frac{\ln \left\|\sigma_{\lambda}\right\|_{X \rightarrow X}}{\ln \lambda} .
$$

In general, $0 \leq \alpha_{X} \leq \beta_{X} \leq 1$. For other properties of symmetric spaces and also the Lorentz and Marcinkiewicz spaces we refer to [2, 25, 27].

Theorem 7 Let $0<\theta<1, X$ be a symmetric space on $(0, \infty)$ and for weights $u$, $v$ on $(0, \infty)$ we have $u(t)^{1 / \theta} v(t)^{1 /(1-\theta)}=1$ for all $t>0$. Consider the sublinear Hardy operator $H^{*} x(t)=x^{* *}(t)=\frac{1}{t} \int_{0}^{t} x^{*}(s) d s$. Then both $H^{*}: X_{u} \rightarrow X_{u}$ and $H^{*}: L_{v}^{\infty} \rightarrow L_{v}^{\infty}$ are bounded if and only if $u(t) \approx v(t) \approx$ constant and $\beta_{X}<1$.

Proof If the Hardy sublinear operator $H^{*}: L_{v}^{\infty} \rightarrow L_{v}^{\infty}$ is bounded, then

$$
\left(\frac{1}{v}\right)^{* *}(t)=\frac{1}{t} \int_{0}^{t}\left(\frac{1}{v}\right)^{*}(s) d s \leq C_{1} \frac{1}{v(t)} \quad \text { for all } t>0 .
$$


From the Semenov imbedding theorem (see [25, Theorem 5.7]),

$$
\sup _{t>0} \frac{\varphi_{X}(t)}{t} \int_{0}^{t} x^{*}(s) d s \leq\|x\|_{X} \quad \text { for all } x \in X
$$

and the boundedness of $H^{*}: X_{u} \rightarrow X_{u}$, we obtain that

$$
\sup _{t>0} \frac{\varphi_{X}(t)}{t} \int_{0}^{t}\left(u H^{*} x\right)^{*}(s) d s \leq C_{0}\|x u\|_{X} \quad \text { for all } x \in X
$$

Using the assumption on weights and (10) we have

$$
\begin{aligned}
\int_{0}^{t}\left(u H^{*} x\right)^{*}(s) d s & =\int_{0}^{t}\left(v^{-\frac{\theta}{1-\theta}} H^{*} x\right)^{*}(s) d s \\
& \geq \int_{0}^{t}\left[C_{1}\left(H^{*}\left(\frac{1}{v}\right)\right)^{\frac{\theta}{1-\theta}} H^{*} x\right]^{*}(s) d s \\
& =C_{1} \int_{0}^{t} H^{*} x(s)\left(H^{*}\left(\frac{1}{v}\right)(s)\right)^{\frac{\theta}{1-\theta}} d s
\end{aligned}
$$

from which we obtain

$$
\sup _{t>0} \frac{\varphi_{X}(t)}{t} \int_{0}^{t} H^{*} x(s)\left[H^{*}\left(\frac{1}{v}\right)(s)\right]^{\frac{\theta}{1-\theta}} d s \leq \frac{C_{0}}{C_{1}}\|x u\|_{X} \quad \text { for all } x \in X .
$$

Assume first that $\lim _{t \rightarrow \infty}\left(\frac{1}{v}\right)^{*}(t)=0$.

For any $\varepsilon>0$ we can find a set $A_{\varepsilon} \subset\left\{s>0: \frac{1}{v(s)}<\varepsilon\right\}$ such that its measure $m\left(A_{\varepsilon}\right)=1$. For the functions $x_{\varepsilon}=\chi_{A_{\varepsilon}}$ we have

$$
\begin{aligned}
\sup _{t>0} \frac{\varphi_{X}(t)}{t} \int_{0}^{t} H^{*} x_{\varepsilon}(s) & {\left[H^{*}\left(\frac{1}{v}\right)(s)\right]^{\frac{\theta}{1-\theta}} d s } \\
& \geq \varphi_{X}(1) \int_{0}^{1} \frac{\min \left(s, m\left(A_{\varepsilon}\right)\right)}{s}\left[H^{*}\left(\frac{1}{v}\right)(s)\right]^{\frac{\theta}{1-\theta}} d s \\
& =\varphi_{X}(1) \int_{0}^{1}\left[\frac{1}{s} \int_{0}^{s}\left(\frac{1}{v}\right)^{*}(\xi) d \xi\right]^{\frac{\theta}{1-\theta}} d s \\
& \geq \varphi_{X}(1) \int_{0}^{1}\left(\frac{1}{v}\right)^{*}(s)^{\frac{\theta}{1-\theta}} d s
\end{aligned}
$$

and putting it into (11) we get

$$
\varphi_{X}(1) \int_{0}^{1}\left(\frac{1}{v}\right)^{*}(s)^{\frac{\theta}{1-\theta}} d s \leq \frac{C_{0}}{C_{1}}\left\|u \chi_{A_{\varepsilon}}\right\|_{X}=\frac{C_{0}}{C_{1}}\left\|\frac{1}{v^{\frac{\theta}{1-\theta}}} \chi_{A_{\varepsilon}}\right\|_{X} \leq \frac{C_{0}}{C_{1}} \varepsilon^{\frac{\theta}{1-\theta}} \varphi_{X}(1)
$$

or

$$
\int_{0}^{1}\left(\frac{1}{v}\right)^{*}(s)^{\frac{\theta}{1-\theta}} d s \leq \frac{C_{0}}{C_{1}} \varepsilon^{\frac{\theta}{1-\theta}}
$$


which gives that $\frac{1}{v(s)}=0$ a.e. But this is impossible.

If $\lim _{t \rightarrow \infty}\left(\frac{1}{v}\right)^{*}(t)=c>0$, then by $(10)$

$$
\frac{1}{v(t)} \geq \frac{c}{C_{1}} \quad \text { for all } t>0
$$

which means that $v$ is bounded.

Assume that $\lim _{t \rightarrow 0^{+}}\left(\frac{1}{v}\right)^{*}(t)=\infty$. For any $k, n \in N$ let $A_{k}=\left\{t>0: 2^{k}<\right.$ $\left.u(t) \leq 2^{k+1}\right\}$ and $B_{n}=\left\{t>0:\left(\frac{1}{v}\right)^{*}(t)>2^{n}\right\}$.

Let us choose $k \in N$ such that $m\left(A_{k}\right)>0$. For any $n \in N, n>k$, we can find $D_{n} \subset A_{k}$ such that $0<m\left(D_{n}\right)<m\left(B_{n}\right)$. Putting $x_{n}=\chi_{D_{n}}$ into (11), we obtain the estimates

$$
\begin{aligned}
\sup _{t>0} \frac{\varphi_{X}(t)}{t} \int_{0}^{t} H^{*} x_{n} & {\left[H^{*}\left(\frac{1}{v}\right)(s)\right]^{\frac{\theta}{1-\theta}} d s } \\
& \geq \sup _{t>0} \frac{\varphi_{X}(t)}{t} \int_{0}^{t} H^{*} x_{n}\left[\left(\frac{1}{v}\right)^{*}(s)\right]^{\frac{\theta}{1-\theta}} d s \\
& \geq \frac{\varphi_{X}\left(m\left(D_{n}\right)\right)}{m\left(D_{n}\right)} \int_{0}^{m\left(D_{n}\right)} \frac{\min \left(s, m\left(D_{n}\right)\right)}{s}\left[\left(\frac{1}{v}\right)^{*}(s)\right]^{\frac{\theta}{1-\theta}} d s \\
& =\frac{\varphi_{X}\left(m\left(D_{n}\right)\right)}{m\left(D_{n}\right)} \int_{0}^{m\left(D_{n}\right)}\left[\left(\frac{1}{v}\right)^{*}(s)\right]^{\frac{\theta}{1-\theta}} d s \\
& \geq \varphi_{X}\left(m\left(D_{n}\right)\right)\left(2^{n}\right)^{\frac{\theta}{1-\theta}}
\end{aligned}
$$

and

$$
\left\|x_{n} u\right\|_{X} \leq 2^{k+1}\left\|\chi_{D_{n}}\right\|_{X}=2^{k+1} \varphi_{X}\left(m\left(D_{n}\right)\right),
$$

which give

$$
\left(2^{n}\right)^{\frac{\theta}{1-\theta}} \varphi_{X}\left(m\left(D_{n}\right)\right) \leq 2^{k+1} \varphi_{X}\left(m\left(D_{n}\right)\right)
$$

and we come to a contradiction.

If $\lim _{t \rightarrow 0^{+}}\left(\frac{1}{v}\right)^{*}(t)=c<\infty$, then $\left\|\frac{1}{v}\right\|_{L^{\infty}}=c$, and we obtain estimation of $v$ from below. Hence, the weight $v$ is equivalent to a constant, and so the weight $u$ is also equivalent to a constant, which means that $X_{u}=X$. By using the theorem on boundedness of the $H^{*}$ operator in a symmetric space $X(c f$. [25, Theorem 6.6]) we have that this is equivalent with the condition $\beta_{X}<1$. This result gives also the reverse implication and the proof is complete.

Corollary 9 Let $X$ be a symmetric space on $(0, \infty)$ with $\beta_{X}=1$. Then for every $1<p<\infty$ there are no weights $u$, v such that $H^{*}: L_{v}^{\infty} \rightarrow L_{v}^{\infty}$ is bounded and $H^{*}: X_{u} \rightarrow X_{u}$ is also bounded, and $\left(X_{u}\right)^{1 / p}\left(L_{v}^{\infty}\right)^{1-1 / p}=X^{(p)}$.

Proof Since $\left\|\sigma_{\lambda}\right\|_{X^{(p)} \rightarrow X^{(p)}}=\left\|\sigma_{\lambda}\right\|_{X \rightarrow X}^{1 / p}$ it follows that $\beta_{X^{(p)}}=\frac{1}{p} \beta_{X}<1$ and by using the theorem on boundedness of the $H^{*}$ operator in a symmetric space $X$ (cf. 
[25, Theorem 6.6]) we have that $H^{*}: X^{(p)} \rightarrow X^{(p)}$ is bounded and $H^{*}: X \rightarrow X$ is unbounded.

Assume that for $H^{*}$ we can find weights $u, v$ such that $H^{*}: L_{v}^{\infty} \rightarrow L_{v}^{\infty}$ is bounded and $H^{*}: X_{u} \rightarrow X_{u}$ is also bounded, and $\left(X_{u}\right)^{1 / p}\left(L_{v}^{\infty}\right)^{1-1 / p}=X^{(p)}$.

Since the equality $X^{(p)}=\left(X_{u}\right)^{1 / p}\left(L_{v}^{\infty}\right)^{1-1 / p}$ gives $\left(X_{u}\right)^{1 / p}\left(L_{v}^{\infty}\right)^{1-1 / p}=X^{(p)}=$ $X^{1 / p}\left(L^{\infty}\right)^{1-1 / p}$, by Theorem 3 ,

$$
u^{1 / p} v^{1-1 / p}=u^{1-\theta} v^{\theta} \approx 1 \text { or } u^{\frac{1}{\theta}} v^{\frac{1}{1-\theta}} \approx 1
$$

By Theorem 7 we come to the conclusion that $\beta_{X}<1$, which is a contradiction with the assumption on $X$ that $\beta_{X}=1$.

Corollary 9 gives the following

Remark 3 The Schur test for the sublinear Hardy operator $H^{*}$ in the $X^{(p)}$ spaces with $\beta(X)=1$ through the weighted $X$ and weighted $L^{\infty}$ spaces does not hold even for $\theta=1-1 / p$.

This example of the Hardy positive sublinear operator shows that without any additional assumptions on the operator the factorization theorem through weighted $L^{1}$ and weighted $L^{\infty}$ spaces cannot be true.

\section{On the Failure of the Factorization Theorem for the Volterra Oper- ator in Some Calderón-Lozanovskiĭ Spaces}

We will show here that the factorization theorem of the Rubio de Francia type is not true in general in Calderón-Lozanovskiu spaces for a simple integral operator such as the Volterra operator (sometimes also called the integration operator) $V x(t)=$ $\int_{0}^{t} x(s) d s$.

Let us formulate the main factorization problem: Let $\varphi \in \mathcal{U}$, four weights $u_{0}, u_{1}$, $v_{0}, v_{1}$ and a bounded positive linear (or sublinear) operator

$$
T: \varphi\left(L_{u_{0}}^{1}, L_{u_{1}}^{\infty}\right) \rightarrow \varphi\left(L_{v_{0}}^{1}, L_{v_{1}}^{\infty}\right)
$$

be given. Can we find four weights $w_{0}, w_{1}, h_{0}, h_{1}$ such that

$$
\varphi\left(L_{u_{0}}^{1}, L_{u_{1}}^{\infty}\right)=\varphi\left(L_{w_{0}}^{1}, L_{w_{1}}^{\infty}\right), \varphi\left(L_{v_{0}}^{1}, L_{v_{1}}^{\infty}\right)=\varphi\left(L_{h_{0}}^{1}, L_{h_{1}}^{\infty}\right)
$$

and

$$
T: L_{w_{0}}^{1} \rightarrow L_{h_{0}}^{1}, T: L_{w_{1}}^{\infty} \rightarrow L_{h_{1}}^{\infty}
$$

are bounded?

The answer to this problem is negative already for the Volterra operator. We will find a function $\varphi \in \mathcal{U}$ and four weights $u_{0}, u_{1}, v_{0}, v_{1}$ for which (12) is true for the Volterra operator but it is not possible to find weights satisfying (13) and (14). By the 
interpolation property of the Calderón-Lozanovskil construction we have that the assumptions (13) and (14) imply boundedness in (12).

We need first some lemmas.

Lemma 7 Let $\varphi \in \mathcal{U}$ and weights $u_{0}, u_{1}, v_{0}, v_{1}$ on $(0, \infty)$ be given. For $t>0$ put

$$
w_{0}(t)=\operatorname{ess} \sup _{0<s \leq t} \frac{1}{u_{0}(s)}, w_{1}(t)=\int_{0}^{t} \frac{1}{u_{1}(s)} d s .
$$

If $\varphi\left(w_{0}, w_{1}\right) \in \varphi\left(L_{v_{0}}^{1}, L_{v_{1}}^{\infty}\right)$, then for the Volterra operator $V x(t)=\int_{0}^{t} x(s) d s$ we have

$$
\|V\|_{\varphi\left(L_{u_{0}}^{1}, L_{u_{1}}^{\infty}\right) \rightarrow \varphi\left(L_{v_{0}}^{1}, L_{v_{1}}^{\infty}\right)}<\infty .
$$

Proof If $x \in \varphi\left(L_{u_{0}}^{1}, L_{u_{1}}^{\infty}\right)$ and the norm is $<1$, then

$$
|x| \leq \varphi\left(\left|x_{0}\right|,\left|x_{1}\right|\right), \text { with }\left\|x_{0}\right\|_{L_{u_{0}}^{1}} \leq 1,\left\|x_{1}\right\|_{L_{u_{1}}^{\infty}} \leq 1
$$

Thus, by Lemma 4 and the definition of weights, we have

$$
\begin{aligned}
|V x| \leq V(|x|) & \leq V\left(\varphi\left(\left|x_{0}\right|,\left|x_{1}\right|\right)\right) \leq \varphi\left(V\left(\left|x_{0}\right|\right), V\left(\left|x_{1}\right|\right)\right) \\
& =\varphi\left(\int_{0}^{t} \frac{1}{u_{0}(s)}\left|x_{0}(s)\right| u_{0}(s) d s, \int_{0}^{t}\left|x_{1}(s)\right| d s\right) \\
& \leq \varphi\left(w_{0}(t) \int_{0}^{t}\left|x_{0}(s)\right| u_{0}(s) d s,\left\|x_{1} u_{1}\right\|_{L^{\infty}} \int_{0}^{t} \frac{1}{u_{1}(s)} d s\right) \\
& \leq \varphi\left(w_{0}(t)\left\|x_{0} u_{0}\right\|_{L^{1}}, w_{1}(t)\left\|x_{1} u_{1}\right\|_{L^{\infty}}\right) \leq \varphi\left(w_{0}(t), w_{1}(t)\right) .
\end{aligned}
$$

Hence $V x \in \varphi\left(L_{v_{0}}^{1}, L_{v_{1}}^{\infty}\right)$ and

$$
\|V\|_{\varphi\left(L_{u_{0}}^{1}, L_{u_{1}}^{\infty}\right) \rightarrow \varphi\left(L_{v_{0}}^{1}, L_{v_{1}}^{\infty}\right)} \leq\left\|\varphi\left(w_{0}, w_{1}\right)\right\|_{\varphi\left(L_{v_{0}}^{1}, L_{v_{1}}^{\infty}\right)}<\infty
$$

Let $\psi(t)=\frac{t}{\ln (1+t)}$ for $t>0$. This is a concave function on $(0, \infty)$ with $\lim _{t \rightarrow 0^{+}} \psi(t)=1$. Then the function $\psi$ given by

$$
\psi(s, t)=s \psi(t / s)=\frac{s}{\ln \left(1+\frac{s}{t}\right)}
$$

belongs to $\mathcal{U}$.

Lemma 8 For the function $\psi$ from (15) we can find weights $u_{0}, u_{1}, v_{0}, v_{1}$ on $(0, \infty)$ such that for the Volterra operator $V x(t)=\int_{0}^{t} x(s)$ ds we have

$$
\|V\|_{L_{u_{0}}^{1} \rightarrow L_{v_{0}}^{1}}=\infty,\|V\|_{L_{u_{1}}^{\infty} \rightarrow L_{v_{1}}^{\infty}}<\infty
$$

and

$$
\|V\|_{\psi\left(L_{u_{0}}^{1}, L_{u_{1}}^{\infty}\right) \rightarrow \psi\left(L_{v_{0}}^{1}, L_{v_{1}}^{\infty}\right)}<\infty
$$

Proof Choose weights $u_{1}, v_{0}$ such that 
(i) $\int_{0}^{t} \frac{1}{u_{1}(s)} d s<\infty$ for all $t>0$,

(ii) $\int_{t}^{\infty} v_{0}(s) d s<\infty$ for all $t>0$,

(iii) $\Theta(t):=\int_{0}^{t} \frac{1}{u_{1}(s)} d s \int_{t}^{\infty} v_{0}(s) d s$ is an increasing function and $\lim _{t \rightarrow \infty} \Theta(t)=\infty$ and put

$$
u_{0}(t)=v_{1}(t)=\frac{1}{\Theta(t)} \int_{t}^{\infty} v_{0}(s) d s=\frac{1}{\int_{0}^{t} \frac{1}{u_{1}(s)} d s} .
$$

For general weights $u, v$ the norms of an operator $V$ between weighted $L^{1}$ and weighted $L^{\infty}$ spaces are known:

$$
\|V\|_{L_{u}^{1} \rightarrow L_{v}^{1}}=\operatorname{ess} \sup _{t>0} \frac{1}{u(t)} \int_{t}^{\infty} v(s) d s
$$

and

$$
\|V\|_{L_{u}^{\infty} \rightarrow L_{v}^{\infty}}=\operatorname{ess} \sup _{t>0} v(t) \int_{0}^{t} \frac{1}{u(s)} d s .
$$

In our case of special weights we obtain

$$
\begin{gathered}
\|V\|_{L_{u_{0}}^{1} \rightarrow L_{v_{0}}^{1}}=\operatorname{ess} \sup _{t>0} \frac{1}{u_{0}(t)} \int_{t}^{\infty} v_{0}(s) d s=\text { ess } \sup _{t>0} \Theta(t)=\infty, \\
\|V\|_{L_{u_{1}}^{\infty} \rightarrow L_{v_{1}}^{\infty}}=\text { ess } \sup _{t>0} v_{1}(t) \int_{0}^{t} \frac{1}{u_{1}(s)} d s=1 .
\end{gathered}
$$

Therefore the first two conditions on $V$ are satisfied. To show the third one we use Lemma 7.

Since $u_{0}$ is a decreasing function it follows that

$$
w_{0}(t)=\operatorname{ess} \sup _{0<s \leq t} \frac{1}{u_{0}(s)}=\frac{1}{u_{0}(t)}=\int_{0}^{t} \frac{1}{u_{1}(s)} d s=w_{1}(t)
$$

and so

$$
\psi\left(w_{0}(t), w_{1}(t)\right)=\psi(1,1) w_{1}(t)=\frac{1}{\ln 2} w_{1}(t) .
$$

If $x_{0}(t)>0$ a.e. on $(0, \infty)$, then

$$
\psi\left(\frac{x_{0}(t)}{v_{0}(t)}, \frac{1}{v_{1}(t)}\right)=\frac{x_{0}(t)}{v_{0}(t) \ln \left(1+\frac{x_{0}(t)}{v_{0}(t)} v_{1}(t)\right)} \geq \frac{1}{v_{1}(t)}=w_{1}(t)
$$

Thus,

$$
\psi\left(w_{0}, w_{1}\right)=\frac{1}{\ln 2} w_{1} \leq \psi\left(\frac{x_{0}}{v_{0}}, \frac{1}{v_{1}}\right) \in \psi\left(L_{v_{0}}^{1}, L_{v_{1}}^{\infty}\right)
$$

and, according to Lemma 7 , the operator $V$ is bounded from $\psi\left(L_{u_{0}}^{1}, L_{u_{1}}^{\infty}\right)$ into $\psi\left(L_{v_{0}}^{1}, L_{v_{1}}^{\infty}\right)$. 
As function $x_{0}(t)>0$ for which $\left\|x_{0}\right\|_{L^{1}} \leq 1$ we can take, for example,

$$
x_{0}(t)=\frac{1}{3} \sum_{k=-\infty}^{\infty} \min \left(1,2^{-2 k}\right) \chi_{\left(2^{k}, 2^{k+1}\right)}
$$

Theorem 8 Let $\psi$ and weights $u_{0}, u_{1}, v_{0}, v_{1}$ on $(0, \infty)$ be the same as in Lemma 8. There are no weights $w_{0}, w_{1}, h_{0}, h_{1}$ on $(0, \infty)$ that satisfy

$$
\psi\left(L_{w_{0}}^{1}, L_{w_{1}}^{\infty}\right)=\psi\left(L_{u_{0}}^{1}, L_{u_{1}}^{\infty}\right), \psi\left(L_{h_{0}}^{1}, L_{h_{1}}^{\infty}\right)=\psi\left(L_{v_{0}}^{1}, L_{v_{1}}^{\infty}\right)
$$

and the Volterra operator $V$ is bounded between

$$
V: L_{w_{0}}^{1} \rightarrow L_{h_{0}}^{1} \text { and } V: L_{w_{1}}^{\infty} \rightarrow L_{h_{1}}^{\infty}
$$

Proof Assume conversely that such weights exist. For the function $\psi$ we have

$$
\rho_{\psi}(a)=\limsup _{t \rightarrow \infty} \frac{\psi(a t, 1)}{\psi(t, 1)}=\limsup _{t \rightarrow \infty} \frac{a t \ln (1+t)}{t \ln (1+a t)}=a \text {. }
$$

Therefore, if $\psi\left(L_{w_{0}}^{1}, L_{w_{1}}^{\infty}\right)=\psi\left(L_{u_{0}}^{1}, L_{u_{1}}^{\infty}\right)$, then, by Theorem 4 and Lemma 3 with $\theta=1$, we have $w_{0} \approx u_{0}$. Similarly, since $\psi\left(L_{h_{0}}^{1}, L_{h_{1}}^{\infty}\right)=\psi\left(L_{v_{0}}^{1}, L_{v_{1}}^{\infty}\right)$ then again by, Corollary 4 with $\theta=1$, we have $h_{0} \approx v_{0}$. According to Lemma 8

$$
\|V\|_{L_{w_{0}}^{1} \rightarrow L_{h_{0}}^{1}}=\|V\|_{L_{u_{0}}^{1} \rightarrow L_{v_{0}}^{1}}=\infty
$$

which is a contradiction.

Immediately from Lemma 8 and Theorem 8 we have the following example:

Example 2 Let $\psi(s, t)=\frac{s}{\ln \left(1+\frac{s}{t}\right)}$. We can find weights $u_{0}, u_{1}, v_{0}, v_{1}$ on $(0, \infty)$ such that the factorization theorem for the Volterra operator $V$ from the space $\psi\left(L_{u_{0}}^{1}, L_{u_{1}}^{\infty}\right)$ into $\psi\left(L_{v_{0}}^{1}, L_{v_{1}}^{\infty}\right)$ does not hold.

Remark 4 Theorem 8 and Example 2 are still true if we take any function $\psi \in \mathcal{U}$ which satisfies two conditions:

$$
\lim _{s \rightarrow 0^{+}} \psi(s, 1)=c>0 \quad \text { and } \quad \rho_{\psi}(a)=\limsup _{t \rightarrow \infty} \frac{\psi(a t, 1)}{\psi(t, 1)}=a
$$

for all $a>0$.

The failure of the factorization theorem for the operator $V$ was given for the function $\psi$ with the property that $\lim _{s \rightarrow 0^{+}} \psi(s, 1)=c>0$ (see Corollary 9 and Remark 4). We will also present a result for when the function $\psi$ satisfies $\lim _{s \rightarrow 0^{+}} \psi(s, 1)=0$. It is enough to prove a lemma corresponding to Lemma 8 . 
For fixed $0<\theta<1$, let

$$
\psi_{\theta}(t)= \begin{cases}t^{\theta} & \text { if } 0 \leq t \leq \tau(\theta) \\ \text { linear } & \text { if } \tau(\theta) \leq t \leq 1 \\ \psi(t) & \text { if } t>1\end{cases}
$$

where $\psi(t)=\frac{t}{\ln (1+t)}$ and $\tau=\tau(\theta)$ is a point in $(0,1)$ such that $\psi_{\theta}$ is concave on $(0, \infty)$, i.e., $\psi^{\prime}(1)<\frac{\psi(1)-\tau}{1-\tau}<\theta \tau^{\theta}$. Such a point exists since $\psi^{\prime}(1)=1 / \ln 2-$ $1 /[2(\ln 2)]^{2}<1 / \ln 2=\psi(1)$ and $\lim _{\tau \rightarrow 0^{+}} \frac{\psi(1)-\tau}{1-\tau}=\psi(1), \lim _{\tau \rightarrow 0^{+}} \tau^{\theta-1}=\infty$. Let

$$
\psi_{\theta}(s, t)=t \psi_{\theta}(s / t) \text { with the above function } \psi_{\theta}(t) .
$$

Then $\psi_{\theta} \in \mathcal{U}, \lim _{s \rightarrow 0^{+}} \psi_{\theta}(s, 1)=0$ and $\rho_{\psi_{\theta}}(a)=a$ for all $a>0$.

Lemma 9 Let $0<\theta<1$ be fixed and let the function $\psi_{\theta} \in \mathcal{U}$ be given by (16). Then there exist weights $u_{0}, u_{1}, v_{0}, v_{1}$ on $(0, \infty)$ such that, for the Volterra operator $V x(t)=\int_{0}^{t} x(s) d s$, we have

$$
\|V\|_{L_{u_{0}}^{1} \rightarrow L_{v_{0}}^{1}}=\infty,\|V\|_{L_{u_{1}}^{\infty} \rightarrow L_{v_{1}}^{\infty}}=1
$$

and

$$
\|V\|_{\psi_{\theta}\left(L_{u_{0}}^{1}, L_{u_{1}}^{\infty}\right) \rightarrow \psi_{\theta}\left(L_{v_{0}}^{1}, L_{v_{1}}^{\infty}\right)} \leq 2
$$

Proof For fixed $\alpha \in(0,1)$ define weights $u_{0}, v_{0}$ by

$$
u_{0}(t)=\min (t, \tau(\theta))^{\alpha} \text { and } v_{0}(t)=\tau(\theta)^{1-\alpha} t^{-2} \text {. }
$$

Then

$$
\begin{aligned}
\|V\|_{L_{u_{0}}^{1} \rightarrow L_{v_{0}}^{1}} & =\underset{t>0}{\operatorname{ess} \sup } \frac{1}{u_{0}(t)} \int_{t}^{\infty} v_{0}(s) d s \\
& =\underset{t>0}{\operatorname{ess} \sup }\left[\min (t, \tau(\theta))^{-\alpha} \tau(\theta)^{1-\alpha} t^{-1}\right] \\
& =\underset{t>0}{\operatorname{ess} \sup \max }\left(\left(\frac{\tau(\theta)}{t}\right)^{1-\alpha}, \frac{\tau(\theta)}{t}\right)=\infty .
\end{aligned}
$$

Choose as weight $u_{1}$ a function which satisfies

$$
\int_{0}^{0} \frac{1}{u_{1}(s)} d s \geq \frac{1}{\tau(\theta) u_{0}(t)} \quad \text { for } t \in(0, \tau(\theta)]
$$

and $u_{1}(t)=\tau(\theta)^{-\alpha}$ for $t>\tau(\theta)$. Let

$$
\frac{1}{v_{1}(t)}= \begin{cases}f(t) \int_{0}^{t} \frac{d s}{u_{1}(s)} & \text { if } 0<t \leq \tau(\theta) \\ \int_{0}^{t} \frac{d s}{u_{1}(s)} & \text { if } t>\tau(\theta)\end{cases}
$$


where $f(t)$ is a function that for $0<t \leq \tau(\theta)$ satisfies

$$
f(t) \geq \max \left(\frac{1}{\tau(\theta) v_{0}(t) \int_{0}^{t} u_{1}(s) d s},\left(\frac{v_{0}(t)}{u_{0}(t)}\right)^{\theta /(1-\theta)}\right) .
$$

As $u_{1}$ and $f$ we can take for example, on $(0, \tau(\theta)]$,

$$
u_{1}(t)=t^{1-\alpha} /(\alpha \tau(\theta)) \text { and } f(t)=\tau(\theta)^{\alpha-1} t^{\theta(1-\alpha) /(1-\theta)} .
$$

Then

$$
\begin{aligned}
\|V\|_{L_{u_{1}}^{\infty} \rightarrow L_{v_{1}}^{\infty}} & =\underset{t>0}{\operatorname{ess} \sup v_{1}(t)} \int_{0}^{t} \frac{1}{u_{1}(s)} d s \\
& =\max \left(\operatorname{ess~sup~}_{0<t \leq \tau(\theta)} \frac{1}{f(t)}, 1\right) \\
& \leq \max \left(\tau(\theta)^{\theta /(1-\theta)}, 1\right)=1 .
\end{aligned}
$$

Consider the functions

$$
x_{0}(t)= \begin{cases}\frac{1}{v_{0}(t)} & \text { if } 0<t \leq \tau(\theta), \\ \frac{1}{u_{0}(t)} & \text { if } t>\tau(\theta)\end{cases}
$$

and $x_{1}(t)=\frac{1}{v_{1}(t)}$. Then

$$
\left\|x_{0}\right\|_{L_{v_{0}}^{1}}=\tau(\theta)+1,\left\|x_{1}\right\|_{L_{v_{1}}^{1}}=1
$$

and for $0<t \leq \tau(\theta)$,

$$
\begin{aligned}
\psi_{\theta}\left(\frac{1}{u_{0}(t)}, \int_{0}^{t} \frac{d s}{u_{1}(s)}\right) & =u_{0}(t)^{-\theta}\left(\int_{0}^{t} \frac{d s}{u_{1}(s)}\right)^{1-\theta} \\
& \leq f(t)^{1-\theta} v_{0}(t)^{-\theta}\left(\int_{0}^{t} \frac{d s}{u_{1}(s)}\right)^{1-\theta} \\
& =\psi_{\theta}\left(\frac{1}{v_{0}(t)}, f(t) \int_{0}^{t} \frac{d s}{u_{1}(s)}\right) \\
& =\psi_{\theta}\left(\frac{1}{v_{0}(t)}, \frac{1}{v_{1}(t)}\right)=\psi_{\theta}\left(x_{0}(t), x_{1}(t)\right) .
\end{aligned}
$$

For $t>\tau(\theta)$,

$$
\psi_{\theta}\left(\frac{1}{u_{0}(t)}, \int_{0}^{t} \frac{d s}{u_{1}(s)}\right)=\psi_{\theta}\left(\frac{1}{u_{0}(t)}, \frac{1}{v_{1}(t)}\right)=\psi_{\theta}\left(x_{0}(t), x_{1}(t)\right) .
$$

Thus, by similar considerations as those in Lemma 7, we find that

$$
\|V\|_{\psi_{\theta}\left(L_{u_{0}}^{1}, L_{u_{1}}^{\infty}\right) \rightarrow \psi_{\theta}\left(L_{v_{0}}^{1}, L_{v_{1}}^{\infty}\right)} \leq 1+\tau(\theta) \leq 2
$$


and Lemma 9 is proved.

Analogously as in the proof of Theorem 8 and using instead of Lemma 8 the just proved Lemma 9, we can formulate a similar theorem under the assumption that $\lim _{s \rightarrow 0^{+}} \psi(s, 1)=0$.

Theorem $8^{\prime}$ Let $\psi$ and weights $u_{0}, u_{1}, v_{0}, v_{1}$ on $(0, \infty)$ be the same as in Lemma 9. There are no weights $w_{0}, w_{1}, h_{0}, h_{1}$ on $(0, \infty)$ that satisfy

$$
\psi\left(L_{w_{0}}^{1}, L_{w_{1}}^{\infty}\right)=\psi\left(L_{u_{0}}^{1}, L_{u_{1}}^{\infty}\right), \psi\left(L_{h_{0}}^{1}, L_{h_{1}}^{\infty}\right)=\psi\left(L_{v_{0}}^{1}, L_{v_{1}}^{\infty}\right)
$$

and so that the Volterra operator $V$ is bounded between

$$
V: L_{w_{0}}^{1} \rightarrow L_{h_{0}}^{1} \text { and } V: L_{w_{1}}^{\infty} \rightarrow L_{h_{1}}^{\infty} .
$$

\section{Factorization of the Averaging Operator}

We shall now consider a factorization theorem for the averaging operator on $[0, \infty)$. Let

$$
A_{I} x(t)=\frac{1}{|I|} \int_{I} x(s) d s \chi_{I}(t), \quad \text { where } I=[a, b] \text { with } a, b>0 .
$$

We can easily see that

$$
\left\|A_{I}\right\|_{L_{u}^{1} \rightarrow L_{u}^{1}}=\frac{1}{|I|} \int_{I} u(s) d s \operatorname{ess} \sup _{t \in I} \frac{1}{u(t)}
$$

and

$$
\left\|A_{I}\right\|_{L_{v}^{\infty} \rightarrow L_{v}^{\infty}}=\frac{1}{|I|} \int_{I} \frac{1}{v(s)} d s \operatorname{sss}_{t \in I} \sup v(t) .
$$

We show that the analogue of the factorization theorem of Muckenhoupt's $A_{p}$-condition (cf. [39]) for the operator $A$ and the space $\psi\left(L_{u}^{1}, L_{v}^{\infty}\right)$ does not hold.

Theorem 9 Let $\psi \in U$ be such a function that $\lim _{s \rightarrow 0^{+}} \psi(s, 1)=c>0$ and $\rho_{\psi}(a)=$ $\lim \sup _{t \rightarrow \infty} \frac{\psi(a t, 1)}{\psi(t, 1)}=$ a for all $a>0$. If $u(t)=\sum_{k=0}^{\infty} 2^{k} \chi_{[k, k+1)}(t)$ for $t \geq 0$, then for the averaging operator $A_{I}$ we have

$$
\sup _{I}\left\|A_{I}\right\|_{\psi\left(L_{u}^{1}, L^{\infty}\right) \rightarrow \psi\left(L_{u}^{1}, L^{\infty}\right)}=C<\infty
$$

and there are no weights $w_{0}, w_{1}, h_{0}, h_{1}$ on $(0, \infty)$ that satisfy the conditions

$$
\psi\left(L_{w_{0}}^{1}, L_{w_{1}}^{\infty}\right)=\psi\left(L_{u}^{1}, L^{\infty}\right), \psi\left(L_{h_{0}}^{1}, L_{h_{1}}^{\infty}\right)=\psi\left(L_{u}^{1}, L^{\infty}\right)
$$

with

$$
\sup _{I}\left\|A_{I}\right\|_{L_{w_{0}}^{1} \rightarrow L_{h_{0}}^{1}}<\infty, \sup _{I}\left\|A_{I}\right\|_{L_{w_{1}}^{\infty} \rightarrow L_{h_{1}}^{\infty}}<\infty .
$$


Proof We have

$$
\begin{aligned}
\sup _{I}\left\|A_{I}\right\|_{L_{u}^{1} \rightarrow L_{u}^{1}} & =\frac{1}{|I|} \int_{I} u(s) d s \operatorname{ess} \sup _{t>0} \frac{1}{u(t)} \\
& \geq \sup _{n \in \mathbf{N}} \frac{1}{n} \int_{0}^{n} u(s) d s \operatorname{ess} \sup _{t \in[0, n]} \frac{1}{u(t)}=\sup _{n \in \mathbf{N}} \frac{2^{n}-1}{n}=\infty .
\end{aligned}
$$

Of course, $\sup _{I}\left\|A_{I}\right\|_{L^{\infty} \rightarrow L^{\infty}}=1$. We show that

$$
\sup _{I}\left\|A_{I}\right\|_{\psi\left(L_{u}^{1}, L^{\infty}\right) \rightarrow \psi\left(L_{u}^{1}, L^{\infty}\right)}=C<\infty
$$

If $|I| \leq 1$ and $I \cap[i, i+1) \neq \varnothing$, then $2^{i-1} \leq u(t) \leq 2^{i+1}$ for all $t \in I$ and

$$
\sup _{I}\left\|A_{I}\right\|_{\psi\left(L_{u}^{1}, L^{\infty}\right) \rightarrow \psi\left(L_{u}^{1}, L^{\infty}\right)} \leq 4
$$

If $|I|>1$, then for all $\left\|x_{0}\right\|_{L_{u}^{1}} \leq 1$ and $\left\|x_{1}\right\|_{L^{\infty}} \leq 1$, we have

$$
A_{I}\left(\psi\left(\left|x_{0}(t)\right|,\left|x_{1}(t)\right|\right)\right) \leq \psi\left(A_{I}\left(\left|x_{0}(t)\right|\right), A_{I}\left(\left|x_{1}(t)\right|\right)\right) \leq \psi\left(A_{I}\left(\left|x_{0}(t)\right|\right), 1\right) .
$$

Since $u(t) \geq 1$ it follows that $a=\frac{1}{|I|} \int_{I}\left|x_{0}(s)\right| d s \leq \frac{1}{|I|} \int_{I}\left|x_{0}(s)\right| u(s) d s \leq \frac{1}{|I|}<1$ and so $\psi(a, 1) \leq \psi(1,1)$. Thus, choosing $x_{0}(t)>0$ a.e. we obtain

$$
A_{I}\left(\psi\left(\left|x_{0}(t)\right|,\left|x_{1}(t)\right|\right)\right) \leq \psi(1,1) \leq \frac{\psi(1,1)}{c} \psi\left(\left|x_{0}(t)\right|, 1\right)
$$

which means that

$$
\sup _{I}\left\|A_{I}\right\|_{\psi\left(L_{u}^{1}, L^{\infty}\right) \rightarrow \psi\left(L_{u}^{1}, L^{\infty}\right)} \leq \frac{\psi(1,1)}{c}
$$

for $|I|>1$. Thus $C=\max \left(4, \frac{\psi(1,1)}{c}\right)$.

The rest of the proof is similar to the proof of Theorem 8 .

\section{The Failure for the Hardy Operator of the Factorization and the Schur Lemma in Some Reflexive Orlicz Spaces}

The Schur lemma for an integral operator $K x(t)=\int k(t, s) x(s) d s$ with a positive kernel $k(t, s) \geq 0$ saying that the operator $K$ is bounded in $L^{p}(1<p<\infty)$ if and only if there exists a positive function $u$ such that

$$
K u^{q}(t) \leq C u^{q}(t) \text { and } K^{\prime} u^{p}(t) \leq C u^{p}(t)
$$

where $1 / p+1 / q=1$ and $K^{\prime}$ is the formal associate operator. We can rewrite this in factorization form: there exists a positive function $u$ such that

$$
K: L_{u^{p}}^{1} \rightarrow L_{u^{p}}^{1} \text { and } K: L_{u^{-p^{\prime}}}^{\infty} \rightarrow L_{u^{-p^{\prime}}}^{\infty}
$$


is bounded. The last statement is a factorization theorem for the operator $K$.

Theorem 8 shows that a similar factorization theorem for the Volterra operator between weighted Orlicz spaces $L^{M}$ is not possible, but these Orlicz spaces are not reflexive. We will show below that the classical Hardy operator $H x(t)=\frac{1}{t} \int_{0}^{t} x(s) d s$, which is a bounded operator in any reflexive Orlicz space $L^{M}$, has in some of them, no factorization through weighted $L^{1}$ and weighted $L^{\infty}$ spaces.

Theorem 10 There exist reflexive Orlicz spaces $L^{M}$ on $(0, \infty)$ for which there are no weights $u_{0}, u_{1}, v_{0}, v_{1}$ on $(0, \infty)$ that satisfy

$$
\varphi\left(L_{u_{0}}^{1}, L_{u_{1}}^{\infty}\right)=\varphi\left(L^{1}, L^{\infty}\right)=L^{M}, \varphi\left(L_{v_{0}}^{1}, L_{v_{1}}^{\infty}\right)=\varphi\left(L^{1}, L^{\infty}\right)=L^{M}
$$

and such that the Hardy operator $H$ is bounded between

$$
H: L_{u_{0}}^{1} \rightarrow L_{v_{0}}^{1} \text { and } H: L_{u_{1}}^{\infty} \rightarrow L_{v_{1}}^{\infty}
$$

In particular, the Schur lemma does not hold for the Hardy operator $H$ in some reflexive Orlicz spaces.

Proof Since the Orlicz space $L^{M}$ is reflexive, it follows that the function $M$ and its complementary $M^{*}$ satisfy the $\Delta_{2}$-condition, that is, $M(2 t) \leq C M(t)$ and $M^{*}(2 t) \leq$ $C M^{*}(t)$ for all $t>0$. For a new Orlicz function $M_{1}$ defined by

$$
M_{1}(t)=\int_{0}^{t} \frac{M(s)}{s} d s, \quad t>0
$$

we have that $M_{1}$ is strictly increasing on $(0, \infty)$. Furthermore $M_{1}$ is equivalent to $M$ since $M(t / 2) \leq M_{1}(t) \leq M(t)$ for all $t>0$, and so $L^{M_{1}}=L^{M}$.

The important step now is a construction of the function $M$ or $\varphi$ with $\varphi(t)=$ $\varphi(t, 1)=M^{-1}(t)$ for which $\rho_{\varphi}(a)$ from Theorem 4 is not equivalent to a power function for all $a>0$. Such constructions we will do later on in Examples 2 and 3 but we continue our proof with the function $M$ having such a property.

Assume conversely that we can find weights $u_{0}, u_{1}, v_{0}, v_{1}$ on $(0, \infty)$ that satisfy

$$
\varphi\left(L_{u_{0}}^{1}, L_{u_{1}}^{\infty}\right)=\varphi\left(L^{1}, L^{\infty}\right)=L^{M_{1}}, \varphi\left(L_{v_{0}}^{1}, L_{v_{1}}^{\infty}\right)=\varphi\left(L^{1}, L^{\infty}\right)=L^{M_{1}}
$$

and the Hardy operator $H: L_{u_{0}}^{1} \rightarrow L_{v_{0}}^{1}$ and $H: L_{u_{1}}^{\infty} \rightarrow L_{v_{1}}^{\infty}$ is bounded.

Then neither $u_{1}$ nor $v_{1}$ is equivalent to a constant function. If $u_{1}$ is equivalent to a constant function, then, observing that $\varphi(s, 1)=M_{1}^{-1}(s)$ is strictly increasing, we can use Theorem 4 , which gives that $u_{0}$ is equivalent to a constant function, and consequently the Hardy operator will be bounded in $L^{1}$, which is not the case. Similarly with $v_{1}$.

Assume now that both weights $u_{1}$ and $v_{1}$ are not equivalent to a constant function. Then, again by Theorem 4 , we obtain that

$$
u_{0}^{\theta} u_{1}^{1-\theta} \approx 1 \text { and } v_{0}^{\theta} v_{1}^{1-\theta} \approx 1
$$


where $\theta$ is the number such that $\rho_{\varphi}(a) \approx a^{\theta}$ for all $a>0$. Thus any $\varphi \in \mathcal{U}$ such that $\rho_{\varphi}(a)$ has different indices $\alpha_{\varphi} \neq \beta_{\varphi}$ gives a counterexample.

We now only need to give an example of such a function. We give below three such examples, but the proofs we put into Appendix A at the end of the paper.

Example 3 Let $0<\theta_{0}<\theta_{1}<1$ and $1=a_{1}<a_{2}<a_{3}<\cdots$ be a sequence such that the quotient $\frac{a_{n+1}}{a_{n}}$ is increasing to infinity. Put $\varphi(t)=t^{\theta_{1}}$ for $0 \leq t \leq 1$ and

$$
\varphi(t)= \begin{cases}\left(\frac{t}{a_{2 n-1}}\right)^{\theta_{0}} \varphi\left(a_{2 n-1}\right) & \text { if } a_{2 n-1} \leq t \leq a_{2 n}, \\ \left(\frac{t}{a_{2 n}}\right)^{\theta_{1}} \varphi\left(a_{2 n}\right) & \text { if } a_{2 n} \leq t \leq a_{2 n+1} .\end{cases}
$$

Then $\varphi$ is a quasi-concave function on $(0, \infty)$, i.e., $\varphi$ is increasing and $\frac{\varphi(t)}{t}$ is decreasing on $(0, \infty)$. It is well known that there exists a concave function $\tilde{\varphi}$ such that $\varphi(t) \leq \tilde{\varphi}(t) \leq 2 \varphi(t)$ (see [25]). Moreover,

$$
\rho_{\varphi}(a)=\max \left(a^{\theta_{0}}, a^{\theta_{1}}\right)
$$

for any $a>0$ and the indices are $\alpha_{\varphi}=\theta_{0}, \beta_{\varphi}=\theta_{1}$.

Example 4 For small $\alpha>0$ and $\sqrt{2} \alpha<\theta \leq 1-\sqrt{2} \alpha$ let

$$
\varphi(t)= \begin{cases}t^{\theta} & \text { if } 0 \leq t \leq e, \\ t^{\theta+\alpha \sin (\ln \ln t)} & \text { if } t \geq e\end{cases}
$$

Then $\varphi$ is a quasi-concave function on $(0, \infty), \rho_{\varphi}(a)=\max \left(a^{\theta-\sqrt{2} \alpha}, a^{\theta+\sqrt{2} \alpha}\right)$ and the indices are $\alpha_{\varphi}=\theta-\sqrt{2} \alpha, \beta_{\varphi}=\theta+\sqrt{2} \alpha$.

Example 5 ( $c f$. [35, pp. 93-94] for $t$ near zero) For $k>0$ and $p>\sqrt{2} k+2$ let

$$
M(t)= \begin{cases}t^{p} & \text { if } 0 \leq t \leq e \\ t^{p+k \sin (\ln \ln t)} & \text { if } t \geq e\end{cases}
$$

Then $M$ is a convex increasing function on $(0, \infty), \rho_{M}(a)=\max \left(a^{p-\sqrt{2} k}, a^{p+\sqrt{2} k}\right)$ and the indices are $\alpha_{\varphi}=\theta-\sqrt{2} k, \beta_{\varphi}=\theta+\sqrt{2} k$.

\section{A Proofs of the Statements in Examples 3, 4 and 5}

Proof in Example 3 From the definition of $\varphi$ we have

$$
\varphi(t)= \begin{cases}t^{\theta_{0}}\left(\prod_{k=1}^{n} \frac{a_{2 k-1}}{a_{2 k-2}}\right)^{\theta_{1}-\theta_{0}} & \text { if } a_{2 n-1} \leq t \leq a_{2 n}, \\ t^{\theta_{1}}\left(\prod_{k=1}^{n} \frac{a_{2 k-1}}{a_{2 k}}\right)^{\theta_{1}-\theta_{0}} & \text { if } a_{2 n} \leq t \leq a_{2 n+1},\end{cases}
$$

for $n=1,2, \ldots$, where $a_{0}=a_{1}=1$. 
We show that $\varphi$ is a quasi-concave function on $(0, \infty)$. If either $a_{2 n-1} \leq s<$ $t \leq a_{2 n}$ or $a_{2 n} \leq s<t \leq a_{2 n+1}$, then clearly $\varphi(s)<\varphi(t)$ and $\frac{\varphi(t)}{t}<\frac{\varphi(s)}{s}$. In the remaining case, $a_{2 n-1} \leq s \leq a_{2 n}<t<a_{2 n+1}$, we have

$$
\begin{aligned}
\varphi(s) & =\left(\frac{s}{a_{2 n-1}}\right)^{\theta_{0}} \varphi\left(a_{2 n-1}\right)=s^{\theta_{0}}\left(\prod_{k=1}^{n} \frac{a_{2 k-1}}{a_{2 k-2}}\right)^{\theta_{1}-\theta_{0}} \\
& \leq a_{2 n}^{\theta_{0}}\left(\prod_{k=1}^{n} \frac{a_{2 k-1}}{a_{2 k-2}}\right)^{\theta_{1}-\theta_{0}}<t^{\theta_{1}}\left(\prod_{k=1}^{n} \frac{a_{2 k-1}}{a_{2 k}}\right)^{\theta_{1}-\theta_{0}} \\
& =\left(\frac{t}{a_{2 n}}\right)^{\theta_{1}} \varphi\left(a_{2 n}\right)=\varphi(t)
\end{aligned}
$$

and

$$
\begin{aligned}
\frac{\varphi(t)}{t} & =t^{\theta_{1}-1}\left(\prod_{k=1}^{n} \frac{a_{2 k-1}}{a_{2 k}}\right)^{\theta_{1}-\theta_{0}}<a_{2 n}^{\theta_{1}-1}\left(\prod_{k=1}^{n} \frac{a_{2 k-1}}{a_{2 k}}\right)^{\theta_{1}-\theta_{0}} \\
& \leq s^{\theta_{0}-1}\left(\prod_{k=1}^{n} \frac{a_{2 k-1}}{a_{2 k}}\right)^{\theta_{1}-\theta_{0}}=\frac{\varphi(s)}{s}
\end{aligned}
$$

and this shows that the function $\varphi$ is increasing and $\frac{\varphi(t)}{t}$ is decreasing on $(0, \infty)$. Note that

$$
\varphi_{-}^{\prime}\left(a_{2 n}\right)=\theta_{0} a_{2 n}^{\theta_{0}-1}\left(\prod_{k=1}^{n} \frac{a_{2 k-1}}{a_{2 k}}\right)^{\theta_{1}-\theta_{0}}<\theta_{1} a_{2 n}^{\theta_{1}-1}\left(\prod_{k=1}^{n} \frac{a_{2 k-1}}{a_{2 k}}\right)^{\theta_{1}-\theta_{0}}=\varphi_{+}^{\prime}\left(a_{2 n}\right)
$$

which means that the function $\varphi$ is not concave on $(0, \infty)$.

Let $a>1$. We want to show that

$$
\liminf _{t \rightarrow \infty} \frac{\varphi(a t)}{\varphi(t)}=a^{\theta_{0}} \text { and } \limsup _{t \rightarrow \infty} \frac{\varphi(a t)}{\varphi(t)}=a^{\theta_{1}} .
$$

Consider several cases, where $n=2,3, \ldots$ is arbitrary but fixed:

1. If $t, a t \in\left[a_{2 n}, a_{2 n+1}\right]$, then $\frac{\varphi(a t)}{\varphi(t)}=a^{\theta_{1}} \geq a^{\theta_{0}}$ and so $\lim \sup _{t \rightarrow \infty} \frac{\varphi(a t)}{\varphi(t)} \geq a^{\theta_{1}}$.

2. If $t$, at $\in\left[a_{2 n-1}, a_{2 n}\right]$, then $\frac{\varphi(a t)}{\varphi(t)}=a^{\theta_{0}} \leq a^{\theta_{1}}$ and so $\liminf _{t \rightarrow \infty} \frac{\varphi(a t)}{\varphi(t)} \leq a^{\theta_{0}}$.

3. If $t \in\left(a_{2 n-2 i}, a_{2 n-2 i+1}\right], i=1,2, \ldots, n$ and $a t \in\left[a_{2 n}, a_{2 n+1}\right]$, then

$$
\frac{\varphi(a t)}{\varphi(t)}=a^{\theta_{1}}\left(\frac{\prod_{k=1}^{n} \frac{a_{2 k-1}}{a_{2 k}}}{\prod_{k=1}^{n-i} \frac{a_{2 k-1}}{a_{2 k}}}\right)^{\theta_{1}-\theta_{0}}=a^{\theta_{1}}\left(\prod_{k=n-i+1}^{n} \frac{a_{2 k-1}}{a_{2 k}}\right)^{\theta_{1}-\theta_{0}} \leq a^{\theta_{1}}
$$


and

$$
\begin{aligned}
\frac{\varphi(a t)}{\varphi(t)} & =a^{\theta_{1}}\left(\prod_{k=n-i+1}^{n} \frac{a_{2 k-1}}{a_{2 k}}\right)^{\theta_{1}-\theta_{0}}=a^{\theta_{0}}\left(a \frac{a_{2 n-2 i+1}}{a_{2 n-2 i+2}} \prod_{k=n-i+2}^{n} \frac{a_{2 k-1}}{a_{2 k}}\right)^{\theta_{1}-\theta_{0}} \\
& \geq a^{\theta_{0}}\left(\frac{a t}{a_{2 n-2 i+2}} \prod_{k=n-i+2}^{n} \frac{a_{2 k-1}}{a_{2 k}}\right)^{\theta_{1}-\theta_{0}} \geq a^{\theta_{0}}\left(\frac{a_{2 n}}{a_{2 n-2 i+2}} \prod_{k=n-i+2}^{n} \frac{a_{2 k-1}}{a_{2 k}}\right)^{\theta_{1}-\theta_{0}} \\
& \geq a^{\theta_{0}}\left(\frac{a_{2 n-2 i+3}}{a_{2 n-2 i+2}} \cdot \frac{a_{2 n-2 i+5}}{a_{2 n-2 i+4}} \cdots \frac{a_{2 n-1}}{a_{2 n-2}}\right)^{\theta_{1}-\theta_{0}} \geq a^{\theta_{0}} .
\end{aligned}
$$

4. If $t \in\left[a_{2 n-2 i-1}, a_{2 n-2 i}\right], i=0,1,2, \ldots, n-1$ and $a t \in\left[a_{2 n}, a_{2 n+1}\right]$, then

$$
\begin{aligned}
\frac{\varphi(a t)}{\varphi(t)} & =a^{\theta_{1}} t^{\theta_{1}-\theta_{0}}\left(\frac{\prod_{k=1}^{n} \frac{a_{2 k-1}}{a_{2 k}}}{\prod_{k=1}^{n-i} \frac{a_{2 k-1}}{a_{2 k}} \cdot a_{2 n-2 i}}\right)^{\theta_{1}-\theta_{0}} \\
& =a^{\theta_{1}} t^{\theta_{1}-\theta_{0}}\left(\prod_{k=n-i+1}^{n} \frac{a_{2 k-1}}{a_{2 k}} \frac{1}{a_{2 n-2 i}}\right)^{\theta_{1}-\theta_{0}} \\
& =a^{\theta_{1}}\left(\frac{t}{a_{2 n-2 i}} \prod_{k=n-i+1}^{n} \frac{a_{2 k-1}}{a_{2 k}}\right)^{\theta_{1}-\theta_{0}} \leq a^{\theta_{1}}
\end{aligned}
$$

and

$$
\begin{aligned}
\frac{\varphi(a t)}{\varphi(t)} & =a^{\theta_{1}}\left(\frac{t}{a_{2 n-2 i}} \prod_{k=n-i+1}^{n} \frac{a_{2 k-1}}{a_{2 k}}\right)^{\theta_{1}-\theta_{0}} \\
& =a^{\theta_{0}}\left(\frac{a t}{a_{2 n-2 i}} \cdot \frac{a_{2 n-2 i+1}}{a_{2 n-2 i+2}} \cdot \frac{a_{2 n-2 i+3}}{a_{2 n-2 i+4}} \cdots \frac{a_{2 n-1}}{a_{2 n}}\right)^{\theta_{1}-\theta_{0}} \\
& \geq a^{\theta_{0}}\left(\frac{a_{2 n}}{a_{2 n-2 i}} \cdot \frac{a_{2 n-2 i+1}}{a_{2 n-2 i+2}} \cdot \frac{a_{2 n-2 i+3}}{a_{2 n-2 i+4}} \cdots \frac{a_{2 n-1}}{a_{2 n}}\right)^{\theta_{1}-\theta_{0}} \\
& =a^{\theta_{0}}\left(\frac{a_{2 n-2 i+1}}{a_{2 n-2 i}} \cdot \frac{a_{2 n-2 i+3}}{a_{2 n-2 i+2}} \cdots \frac{a_{2 n-1}}{a_{2 n-2}}\right)^{\theta_{1}-\theta_{0}} \geq a^{\theta_{0}}
\end{aligned}
$$

5. If $t \in\left[a_{2 n-2 i}, a_{2 n-2 i+1}\right], i=1,2, \ldots, n$, and $a t \in\left[a_{2 n-1}, a_{2 n}\right]$, then

$$
\begin{aligned}
\frac{\varphi(a t)}{\varphi(t)} & =a^{\theta_{0}} t^{\theta_{0}-\theta_{1}} \frac{\left(\frac{a_{1} \cdot a_{3} \cdots a_{2 n-1}}{a_{0} \cdot a_{2} \cdots a_{2 n-2}}\right)^{\theta_{1}-\theta_{0}}}{\left(\frac{a_{1} \cdot a_{3} \cdots a_{2 n-2 i-1}}{a_{0} \cdot a_{2} \cdots \cdot a_{2 n-2 i}}\right)^{\theta_{1}-\theta_{0}}} \\
& =a^{\theta_{0}} t^{\theta_{0}-\theta_{1}}\left(\frac{a_{2 n-2 i+1}}{a_{2 n-2 i+2}} \cdot \frac{a_{2 n-2 i+3}}{a_{2 n-2 i+4}} \cdots \frac{a_{2 n-3}}{a_{2 n-2}} \cdot a_{2 n-1}\right)^{\theta_{1}-\theta_{0}} \\
& =a^{\theta_{0}}\left(\frac{a_{2 n-2 i+1}}{t} \cdot \frac{a_{2 n-2 i+2}}{a_{2 n-2 i+3}} \cdots \frac{a_{2 n-1}}{a_{2 n-2}}\right)^{\theta_{1}-\theta_{0}} \geq a^{\theta_{0}}
\end{aligned}
$$


and

$$
\begin{aligned}
\frac{\varphi(a t)}{\varphi(t)} & =a^{\theta_{0}} t^{\theta_{0}-\theta_{1}}\left(\frac{a_{2 n-2 i+1}}{a_{2 n-2 i+2}} \cdot \frac{a_{2 n-2 i+3}}{a_{2 n-2 i+4}} \cdots \frac{a_{2 n-3}}{a_{2 n-2}} \cdot a_{2 n-1}\right)^{\theta_{1}-\theta_{0}} \\
& =a^{\theta_{0}}\left(\frac{a_{2 n-1}}{t} \cdot \frac{a_{2 n-2 i+1}}{a_{2 n-2 i+2}} \cdot \frac{a_{2 n-2 i+3}}{a_{2 n-2 i+4}} \cdots \frac{a_{2 n-3}}{a_{2 n-2}}\right)^{\theta_{1}-\theta_{0}} \leq a^{\theta_{1}} .
\end{aligned}
$$

6. If $t \in\left[a_{2 n-2 i-1}, a_{2 n-2 i}\right], i=1,2, \ldots, n-1$, and $a t \in\left[a_{2 n-1}, a_{2 n}\right]$, then

$$
\begin{aligned}
\frac{\varphi(a t)}{\varphi(t)} & =a^{\theta_{0}}\left(\frac{\prod_{k=1}^{n} \frac{a_{2 k-1}}{a_{2 k-2}}}{\prod_{k=1}^{n-i} \frac{a_{2 k-1}}{a_{2 k-2}}}\right)^{\theta_{1}-\theta_{0}} \\
& =a^{\theta_{0}}\left(\prod_{k=n-i+1}^{n} \frac{a_{2 k-1}}{a_{2 k-2}}\right)^{\theta_{1}-\theta_{0}} \geq a^{\theta_{0}}
\end{aligned}
$$

and

$$
\begin{aligned}
\frac{\varphi(a t)}{\varphi(t)} & =a^{\theta_{0}}\left(\frac{a_{2 n-2 i+1} \cdot a_{2 n-2 i+3} \cdots a_{2 n-1}}{a_{2 n-2 i} \cdot a_{2 n-2 i+2} \cdots a_{2 n-2}}\right)^{\theta_{1}-\theta_{0}} \\
& =a^{\theta_{0}}\left(\frac{a_{2 n-1}}{a_{2 n-2 i}} \cdot \frac{a_{2 n-2 i+1}}{a_{2 n-2 i+2}} \cdot \frac{a_{2 n-2 i+1}}{a_{2 n-2 i+2}} \cdots \frac{a_{2 n-3}}{a_{2 n-2}}\right)^{\theta_{1}-\theta_{0}} \\
& \leq a^{\theta_{0}}\left(\frac{a_{2 n-1}}{a_{2 n-2 i}}\right)^{\theta_{1}-\theta_{0}} \leq a^{\theta_{0}}\left(\frac{a t}{t}\right)^{\theta_{1}-\theta_{0}}=a^{\theta_{1}} .
\end{aligned}
$$

From all these cases we see that (17) is true and the proof of Lemma 2 is complete.

Proof in Example 4 The function $\varphi$ is quasi-concave on $(0, \infty)$. It is enough to see that for $t \geq e$,

$$
\varphi^{\prime}(t)=\frac{\varphi(t)}{t}[\theta+\alpha(\sin \ln \ln t+\cos \ln \ln t)]=\frac{\varphi(t)}{t}\left[\theta+\sqrt{2} \alpha \sin \left(\ln \ln t+\frac{\pi}{4}\right)\right]
$$

and

$$
(\varphi(t) / t)^{\prime}=\frac{\varphi(t)}{t}\left[\theta-1+\sqrt{2} \alpha \sin \left(\ln \ln t+\frac{\pi}{4}\right)\right] .
$$

We show now that $\rho_{\varphi}(a)=\max \left(a^{\theta-\sqrt{2} \alpha}, a^{\theta-\sqrt{2} \alpha}\right)$. If $a>1$ and $t>e$, then

$$
\begin{aligned}
\frac{\varphi(a t)}{\varphi(t)} & =a^{\theta+\alpha \sin (\ln \ln (a t))} t^{\alpha[\sin (\ln \ln (a t))-\sin (\ln \ln t)]} \\
& =a^{\theta+\alpha \sin (\ln \ln (a t))} t^{2 \alpha \sin [(\ln \ln (a t)-\ln \ln t) / 2] \cos [(\ln \ln (a t)+\ln \ln t) / 2]} \\
& =a^{\theta+\alpha \sin [\ln \ln (a t)]} t^{2 \alpha \sin [\ln (1+\ln a / \ln t) / 2] \cos [\ln \ln t+\ln (1+\ln a / \ln t) / 2]} .
\end{aligned}
$$

Since for $|u| \leq \frac{1}{2}$ we have $\ln (1+u)=u b(u)$ where $|b(u)| \leq 2$ and by the Lagrange mean-value theorem,

$$
\cos (x+h)=\cos x+c(x, h) h \text { with }|c(x, h)| \leq 1,
$$


it follows that for large $t$ and all $a>1$,

$$
\begin{aligned}
\cos \left[\ln \ln t+\frac{1}{2} \ln \left(1+\frac{\ln a}{\ln t}\right)\right] & =\cos \left[\ln \ln t+\frac{1}{2} \frac{\ln a}{\ln t} b(a, t)\right] \\
& =\cos (\ln \ln t)+\frac{1}{2} \frac{\ln a}{\ln t} b(a, t) c(a, t) \\
& =\cos (\ln \ln t)+\frac{1}{2} \frac{\ln a}{\ln t} d(a, t)
\end{aligned}
$$

where $|d(a, t)| \leq 2$. Thus

$$
\begin{aligned}
\frac{\varphi(a t)}{\varphi(t)} & =a^{\theta+\alpha \sin [\ln \ln (a t)]} t^{2 \alpha \sin ((1+\ln a / \ln t) / 2[\cos [\ln \ln t)+d(a, t) \ln a / 2 \ln t]} \\
& =a^{\theta+\alpha \sin [\ln \ln (a t)]+2 \alpha \frac{\ln t}{\ln a} \sin \sin ((1+\ln a / \ln t) / 2[\cos [\ln \ln t)+d(a, t) \ln a / 2 \ln t]} .
\end{aligned}
$$

Since

$$
\lim _{t \rightarrow \infty} \frac{\ln t}{\ln a} 2 \sin \frac{\ln \left(1+\frac{\ln a}{\ln t}\right)}{2}=1
$$

it follows that

$$
\frac{\varphi(a t)}{\varphi(t)}=a^{\theta+\alpha \sin [\ln \ln (a t)]+\alpha \cos [\ln \ln (a t)]+e(a, t)},
$$

where $\lim _{t \rightarrow \infty} e(a, t)=0$ and so

$$
\begin{aligned}
\limsup _{t \rightarrow \infty} \frac{\varphi(a t)}{\varphi(t)} & =a^{\theta+\alpha \lim \sup _{t \rightarrow \infty}\{\sin [\ln \ln (a t)]+\cos [\ln \ln (a t)]\}} \\
& =a^{\theta+\alpha \lim \sup _{u \rightarrow \infty}(\sin u+\cos u)}=a^{\theta+\sqrt{2} \alpha}
\end{aligned}
$$

and

$$
\liminf _{t \rightarrow \infty} \frac{\varphi(a t)}{\varphi(t)}=a^{\theta-\sqrt{2} \alpha}
$$

The proof is complete.

Proof in Example 5 For $t \geq e$ we have

$$
M^{\prime}(t)=\frac{M(t)}{t}\left[p+\sqrt{2} p \sin \left(\ln \ln t+\frac{\pi}{4}\right)\right],
$$

and

$$
\begin{aligned}
M^{\prime \prime}(t)=\frac{M(t)}{t^{2}} & \left\{\left[p-1+\sqrt{2} k \sin \left(\ln \ln t+\frac{\pi}{4}\right)\right]\left[p-1+\sqrt{2} k \sin \left(\ln \ln t+\frac{\pi}{4}\right)\right]\right. \\
& \left.-\frac{\sqrt{2} k}{\ln t} \sin \left(\ln \ln t-\frac{\pi}{4}\right)\right\} .
\end{aligned}
$$

Also $M^{\prime}\left(e^{-}\right)=p e^{p-1} \leq(p+k) e^{p-1}=M^{\prime}\left(e^{-}\right)$. Therefore we can show that $M$ is increasing, convex on $(0, \infty)$ and the proof of the fact that $\rho_{M}(a)=\max \left(a^{\theta-\sqrt{2} k}\right.$, $\left.a^{\theta-\sqrt{2} k}\right)$ for all $a>0$ is similar to Example 3.

Acknowledgement The authors are grateful to the anonymous referee for careful reading of the paper and various valuable comments, suggestions and improvements. 


\section{References}

[1] I. Asekritova and N. Krugljak, On equivalence of $K$ - and J-methods for $(n+1)$-tuples of Banach spaces. Studia Math. 122(1997), 99-116.

[2] C. Bennett and R. Sharpley, Interpolation of Operators. Academic Press, Boston 1988.

[3] E. I. Berezhnoŭ, Interpolation of positive operators in the spaces $\varphi\left(X_{0}, X_{1}\right)$. In: Qualitative and Approximate Methods for the Investigation of Operator Equations, Yaroslav. Gos. Univ., Yaroslavl 1981, pp. 3-12 (Russian).

[4] Theorems on the representation of spaces and Schur's lemma. Dokl. Akad. Nauk 344(1995), 727-729; English Transl. in Doklady Math. 52(1995), 252-254.

[5] An inverse problem in the theory of the interpolation of operators. Mat. Zametki 59(1996), 323-333; English Transl. in Math. Notes 59(1996), 227-233.

[6] E. I. Berezhnoĭ and M. Mastyło, On Calderón-Lozanovskĭ construction. Bull. Polish Acad. Sci. Math. 37(1989), 23-33.

[7] The Lions problem for Gustavson-Peetre functor. Publ. Math. 34(1990), 175-180.

[8] J. Bergh and J. Löfström, Interpolation Spaces. Springer, Berlin 1976.

[9] S. Bloom, Solving weighted norm inequalities using the Rubio de Francia algorithm. Proc. Amer. Math. Soc. 101(1987), 306-312.

[10] Yu. A. Brudnyı and N. Ya. Krugljak, Interpolation Functors and Interpolation Spaces I. North-Holland, Amsterdam 1991.

[11] A. P. Calderón, Intermediate spaces and interpolation, the complex method. Studia Math. 24(1964), 113-190.

[12] M. Christ, Weighted norm inequalities and Schur's lemma. Studia Math. 78(1984), 309-319.

[13] R. Coifman, P. W. Jones, and J. L. Rubio de Francia, Constructive decomposition of BMO functions and factorization of $A_{p}$ weights. Proc. Amer. Math. Soc. 87(1983), 675-676.

[14] M. Cwikel, P. G. Nilsson and G. Schechtman, Interpolation of Weighted Banach Lattices/ A Characterization of Relatively Decomposable Banach Lattices. Mem. Amer. Math. Soc. 787, 2003.

[15] E. Gagliardo, On integral transformations with positive kernel. Proc. Amer. Math. Soc. 16(1965), 429-434.

[16] J. Garcia-Cuerva and J. Rubio de Francia, Weighted Norm Inequalities and Related Topics. North-Holland, Amsterdam, 1985.

[17] E. Hernández, Factorization and extrapolation of pairs of weights. Studia Math. 45(1989), 179-193.

[18] W Weighted inequalities through factorization. Publ. Math. 35(1991), 141-153.

[19] S. Janson, P. Nilsson, and J. Peetre, Notes on Wolff's note on interpolation spaces. Proc. London Math. Soc. 48(1984), 283-299.

[20] G. B. Jawerth, Weighted inequalities for maximal operators: linearization, localization and factorization. Amer. J. Math. 108(1986), 361-414.

[21] P. Jones, Factorization of $A_{p}$-weights. Ann. of Math. 111(1980), 511-530.

[22] A. Kamińska, L. Maligranda, and L. E. Persson, Indices, convexity and concavity of Calderón-Lozanovskiu spaces. Math. Scand. 92(2003), 141-160.

[23] V. B. Korotkov, Integral Operators. Nauka Sibirsk. Otdel., Novosibirsk, 1983, (Russian).

[24] M. Krbec and L. Pick, On imbeddings between weighted Orlicz spaces. Z. Anal. Anwendungen 10(1991), 107-117.

[25] S. G. Krein, Yu. I. Petunin, and E. M. Semenov, Interpolation of Linear Operators. Nauka, Moscow, 1978; Translations of Mathematical Monographs 54, American Mathematical Society, Providence, RI, 1982.

[26] N. Krugljak and L. Maligranda, Calderón-Lozanovskiu construction on weighted Banach function lattices. J. Math. Anal. Appl. 288(2003), 744-757.

[27] J. Lindenstrauss and L. Tzafriri, Classical Banach Spaces, II. Function Spaces. Springer-Verlag, Berlin, New York, 1979.

[28] J. L. Lions and E. Magenes, Problèmes aux Limites Non Homogènes et Applications I. Springer, Berlin, 1972.

[29] G. Ya. Lozanovskiǔ, On some Banach lattices. Sibirsk. Mat. Z. 10(1969), 584-599; English Transl. in Siberian. Math. J. 10(1969), 419-431.

[30] — On some Banach lattices IV. Sibirsk. Mat. Z. 14(1973), 140-155; English Transl. in Siberian. Math. J. 14(1973), 97-108.

[31] Transformations of ideal Banach spaces by means of concave functions. In: Qualitative and Approximate Methods for the Investigation of Operator Equations, Yaroslav. Gos. Univ., Yaroslavl' 1978, pp. 122-147 (Russian).

[32] L. Maligranda, Calderón-Lozanovskiu spaces and interpolation of operators. Semesterbericht Funktionalanalysis, Tübingen 8(1985), 83-92. 
[33] On commutativity of interpolation with intersection. Rend. Circ. Mat. Palermo 10(1985), $113-118$.

[34] A property of interpolation spaces. Arch. Math. 48(1987), 82-84.

[35] Orlicz Spaces and Interpolation. Sem. Math. 5, Univ. of Campinas, Campinas SP, Brazil, 1989.

[36] Why Hölder's inequality should be called Rogers' inequality. Math. Inequal. Appl. 1(1998), 69-83.

[37] Positive bilinear operators in Calderón-Lozanovskiŭ spaces. Arch. Math. 81(2003), 26-37.

[38] L. Maligranda and L. E. Persson, Generalized duality of some Banach function spaces. Indag. Math. 51(1989), 323-338.

[39] B. Muckenhoupt, Weighted norm inequalities for the Hardy maximal function. Trans. Amer. Math. Soc. 165(1972), 207-226.

[40] J. Musielak, Orlicz Spaces and Modular Spaces. Lecture Notes in Math. 1034, Springer-Verlag, Berlin, 1983.

[41] P. Nilsson, Reiteration theorems for real interpolation and approximation spaces. Ann. Mat. Pura Appl. 132(1982), 291-330.

[42] Interpolation of Banach lattices. Studia Math. 82(1985), 135-154.

[43] V. I. Ovchinnikov, Interpolation theorems that arise from Grothendieck's inequality. Funktsional. Anal. i Prilozhen. (4) 10(1976), 45-54; English transl. in Functional Anal. Appl. 10(1976), 287-294 (1977).

[44] The Methods of Orbits in Interpolation Theory. Math. Reports 1, Part 2, Harwood Academic Publishers 1984, 349-516.

[45] G. Pisier, Some applications of the complex interpolation method to Banach lattices. J. Analyse Math. 35(1979), 264-281.

[46] S. Reisner, Some remarks on Lozanovskyi's intermediate normed lattices. Bull. Polish Acad. Sci. Math. 41(1993), 189-196 (1994).

[47] R. Rochberg, Function theoretic results for complex interpolation families of Banach spaces. Trans. Amer. Math. Soc. 284(1984), 745-758.

[48] J. L. Rubio de Francia, A new technigue in the theory of $A_{p}$-weights. In: Topics in Modern Harmonic Analysis, Roma, 1983, pp. 571-579.

[49] Factorization theory and $A_{p}$-weights. Amer. J. Math. 106(1984), 533-547.

[50] V. A. Shestakov, Transformations of Banach ideal spaces and interpolation of linear operators. Bull. Acad. Polon. Sci. Sér/ Math. 29(1981), 569-577 (1982) (Russian).

[51] J. D. Stafney, Analytic interpolation of certain multiplier spaces. Pacific J. Math. 32(1970), 241-248.

[52] P. Szeptycki, Notes on Integral Transformations. Dissertationes Math. (Rozprawy Mat.) 231, 1984.

[53] H. Triebel, Interpolation Theory, Function Spaces, Differential Operators. VEB Deutscher Verlag der Wissenschaften, Berlin, 1978.

[54] R. Wallstén, Remarks on interpolation of subspaces. In: Function Spaces and Applications, Lecture Notes in Math. 1302, Springer, Berlin, 1988, pp. 410-419.

Department of Mathematics

Yaroslavl' State University

Sovetskaya 14

150000 Yaroslavl'

Russia

email: ber@uniyar.ac.ru
Department of Mathematics

Luleå University of Technology

SE-971 87 Luleå

Sweden

email: lech@sm.luth.se

website: www.sm.luth.se/ lech/ 